Submitted to the IEEE Trans. Antennas Propagation; April 22, 1999; revised April 6, 2001

\title{
Application of Haar-Wavelet Based Multiresolution Time-Domain Schemes to Electromagnetic Scattering Problems
}

\author{
Traian Dogaru and Lawrence Carin \\ Department of Electrical and Computer Engineering \\ Duke University \\ Box 90291
}

Durham, NC 27708-0291

\begin{abstract}
The multiresolution time-domain (MRTD) algorithm is applied to the problem of general two-dimensional electromagnetic scattering. A Haar wavelet expansion is utilized. A parallel between Haar MRTD and the classic Yee finite difference time-domain (FDTD) algorithm is discussed, and results of simulations on canonical targets are shown for comparison. We focus on the incident-field implementation, which, in our case, consists of a pulsed plane wave. Also, we consider scattering in a half-space environment, with application to subsurface sensing. The results illustrate the advantage of the Haar MRTD method as compared with the classic FDTD, which consists of reduced memory and execution time requirements, without sacrificing accuracy.
\end{abstract}

\section{Introduction}

During the last two decades, the Finite-Difference Time-Domain (FDTD) algorithm has been used extensively in modeling a large variety of electromagnetic field problems [1-11], including scattering by various targets in general environments. The method allows the modeling of practically arbitrary inhomogeneities, while remaining simple and computationally efficient. However, the main limitation of the classic FDTD scheme is numerical dispersion, which makes it difficult to run simulations over an electrically large domain without introducing significant errors [1]. Efforts have been made to ameliorate the numerical-dispersion problem. Some of the 
solutions use higher-order finite-difference schemes [2,3]. However, these methods often have difficulties in modeling general media configurations. Another solution to the dispersion error issue was suggested in $[4,5]$ and was termed the pseudospectral time-domain (PSTD) method.

A promising approach to this problem, introduced in [12], is called the multiresolution time-domain (MRTD) method. In this approach, Maxwell's equations are discretized using a method-of-moments procedure [13], instead of approximating the partial derivatives with finite differences. It can be shown [14] that an expansion of the fields using rectangular-pulse basis functions in both time and space leads to the classic Yee algorithm. However, one can employ any other set of complete basis functions. The use of wavelets in this situation is very appealing, because it allows a multiresolution analysis [12,15-19] to be applied across the computational domain, thereby reducing the number of field coefficients needed. In particular, higher resolution is introduced in the zones with faster spatial variation of the fields, while keeping a lower-resolution discretization in the slowly varying regions, without a significant loss in accuracy. This approach can be related to data compression techniques [20], in which the wavelet basis functions have proven extremely useful for a large number of applications. The MRTD technique has been employed previously in modeling microwave circuits [15]. In this paper, we demonstrate the application of the technique to scattering problems.

Choosing the most appropriate scaling and wavelet functions for a given application is a central issue in multiresolution analysis. The authors of [12] proposed the use of cubic spline Battle-Lemarie scaling and wavelet functions [20] in the spatial coordinates (while keeping rectangular-pulse basis functions for time). These wavelet and scaling functions have nice properties in terms of smoothness, and finite support in the spectral domain. However, they do not have finite support in the spatial domain, which implies that a large number of adjacent field coefficients need be considered when updating the fields at a given point. The problem becomes more dramatic with the introduction of higher-level wavelet functions, especially in regions with material inhomogeneities (in which higher resolution is primarily needed). Also, in the case of scattering problems in two or three dimensions, the implementation of the incident field as a 
plane wave as well as the perfectly matched layer (PML) absorbing boundary condition (ABC) become complicated, vitiating the benefits of MRTD, in terms of computational resources.

In order to apply the multiresolution technique, while preserving relative algorithmic simplicity, we apply the Haar scaling and wavelet functions [20]. These constitute the simplest family of scaling and wavelet functions, and their finite temporal/spatial support significantly aids MRTD implementation. For example, this property makes implementation of an incident plane-wave field relatively easy. Further, material inhomogenenities (including the PML) can be handled simply with the Haar basis. Moreover, it will be shown that an MRTD scheme using $n$ Haar wavelet levels yields a set of equations which is equivalent to the classic Yee scheme, using $2^{n}$ more dense samples. A detailed account of stability and dispersion analysis for the BattleLemarie MRTD schemes is given in [16]. However, based on the equivalence between the Haar MRTD and Yee FDTD schemes, we can make use of the classic results on stability and numerical dispersion established for the latter when analyzing the former scheme.

Before we proceed with the description of our Haar MRTD formulation, we acknowledge the work of other authors in this area [17,18]. In these previous papers, a Haar-basis-function field expansion was employed and the connection with the Yee FDTD scheme was made. However, a closer inspection of the MRTD equations obtained in $[17,18]$ demonstrates that the coefficients representing the fields at one resolution level are de-coupled from those representing the fields at another level of resolution. Different levels of resolutions can be coupled only through certain types of boundaries [17]. However, there are situations when it is impossible to obtain a true multiresolution structure of the computational domain. This happens, for instance, in the case of dielectric inhomogeneities conformal to the grid, as shown in Sec. IV.A. Our formulation of the Haar MRTD algorithm is different from [17,18], it allowing coupling between the scaling and wavelet components in the field equations. This is obtained by a different staggering scheme (vis-a-vis $[17,18]$ ) between the support of the electric and magnetic field basis functions (see Sec. II). The equivalence with the classic Yee FDTD scheme can still be rigorously established. Moreover, the scheme can be consistently extended to an arbitrary 
number of wavelet levels (see Sec. II.B).

The Haar MRTD algorithm allows us to formulate a numerical scheme that resembles the original FDTD method, applied to a multi-grid structure of the computational domain (different spatial resolutions applied locally). Most of the multi-grid FDTD algorithms [6-8] compute fields

at points situated on a fine mesh, by interpolation schemes involving the fields at the nodes of the main (coarse) mesh. Although these methods have been applied successfully to particular electromagnetic wave problems, some of them employ empirically derived adjustments in their formulations, in order to circumvent instability problems. While these may work well in certain situations, it is not clear whether they can be applied generally. However, the MRTD technique offers a completely different and rigorous approach to this problem, within a stable algorithmic construction.

In this paper, we first establish the MRTD equations for a homogeneous region using the Haar scaling and wavelet functions and make correspondences with the Yee scheme (Section II). In Section III we discuss the implementation of the incident fields as a plane wave and extend the formulation to inhomogeneous dielectric (lossy) materials. In Section IV, we show the results of simulations on some canonical targets and evaluate the accuracy of the method as compared to the classic FDTD. Conclusions follow in Section V.

\section{The Haar-Wavelet MRTD Scheme}

\section{A. One-dimensional MRTD using one level Haar wavelet functions}

First we consider a homogeneous medium. Since the most general notations involve a large number of indices and are difficult to read, we present the MRTD equations in one dimension to begin with, and then extend them to two dimensions (further extension to three dimensions is also straightforward). The prototype Haar scaling and wavelet functions (up to two levels) [20] are shown in Fig. 1. For time discretization we use rectangular pulse functions which we call $h(t)$ (identical to the scaling function $\Phi^{0}(x)$, but we introduce separate notations to 
distinguish between the coordinates). The shifted versions of these basis functions are:

$$
\begin{aligned}
& h_{k}(t)=h\left(\frac{t}{\Delta t}-k\right) \\
& \Phi_{m}^{0}(x)=\Phi^{0}\left(\frac{x}{\Delta x}-m\right) \\
& \Psi_{m}(x)=\Psi\left(\frac{x}{\Delta x}-m\right)
\end{aligned}
$$

where $k$ and $m$ are integers, whereas $\Delta x$ and $\Delta t$ are the spatial and temporal discretization steps, respectively (the grid is considered uniform in both space and time). In (3), we do not mention a particular wavelet order, since the equation is valid at all levels.

Maxwell's equations for one-dimensional propagation in the $x$ direction are:

$$
\begin{aligned}
& \frac{\partial E_{z}}{\partial t}=\frac{1}{\varepsilon} \frac{\partial H_{y}}{\partial x} \\
& \frac{\partial H_{y}}{\partial t}=\frac{1}{\mu} \frac{\partial E_{z}}{\partial x}
\end{aligned}
$$

It is easy to see that using Haar scaling functions alone (which are in fact rectangular pulses) for field expansion and applying the method of moments, we obtain exactly the classic FDTD equations. Therefore, we start with a field expansion which includes scaling functions and one level of wavelet functions:

$$
\begin{aligned}
& E_{z}(x, t)=\sum_{k, m=-\infty}^{\infty}\left[E_{k, m}^{\Phi^{0}} h_{k}(t) \Phi_{m}^{0}(x)+E_{k, m}^{\Psi^{0}} h_{k}(t) \Psi_{m}^{0}(x)\right] \\
& H_{y}(x, t)=\sum_{k, m=-\infty}^{\infty}\left[H_{k, m}^{\Phi^{0}} h_{k+1 / 2}(t) \Phi_{m+1 / 4}^{0}(x)+H_{k, m}^{\Psi^{0}} h_{k+1 / 2}(t) \Psi_{m+1 / 4}^{0}(x)\right]
\end{aligned}
$$


Figure 2 indicates that the support of the scaling/wavelet functions corresponding to the electric and magnetic field are displaced by one quarter of a cell, with respect to the other. This is different from the scheme described in $[17,18]$, where the shift between the electric and magnetic field basis function is half of a cell (the latter yielding decoupled scaling and wavelet coefficients). After Galerkin sampling in space and time [13], one obtains the following MRTD equations:

$$
\begin{aligned}
& E_{k+1, m}^{\Phi^{0}}=E_{k, m}^{\Phi^{0}}+\frac{\Delta t}{\varepsilon \Delta x}\left(H_{k, m}^{\Phi^{0}}-H_{k, m}^{\Psi^{0}}-H_{k, m-1}^{\Phi^{0}}+H_{k, m-1}^{\Psi^{0}}\right) \\
& E_{k+1, m}^{\Psi^{0}}=E_{k, m}^{\Psi^{0}}+\frac{\Delta t}{\varepsilon \Delta x}\left(H_{k, m}^{\Phi^{0}}+3 H_{k, m}^{\Psi^{0}}-H_{k, m-1}^{\Phi^{0}}+H_{k, m-1}^{\Psi^{0}}\right) \\
& H_{k, m}^{\Phi^{0}}=H_{k-1, m}^{\Phi^{0}}+\frac{\Delta t}{\mu \Delta x}\left(E_{k, m+1}^{\Phi^{0}}+E_{k, m+1}^{\Psi^{0}}-E_{k, m}^{\Phi^{0}}-E_{k, m}^{\Psi^{0}}\right) \\
& H_{k, m}^{\Psi^{0}}=H_{k-1, m}^{\Psi^{0}}+\frac{\Delta t}{\mu \Delta x}\left(-E_{k, m+1}^{\Phi^{0}}-E_{k, m+1}^{\Psi^{0}}+E_{k, m}^{\Phi^{0}}-3 E_{k, m}^{\Psi^{0}}\right)
\end{aligned}
$$

It is interesting to note that these equations are equivalent to the Yee FDTD equations obtained for a grid with step $\Delta x / 2$. To illustrate this, Fig. 3 depicts the support of the electric field expansion terms according to the two schemes (the index $Y$ stands for Yee). The amplitudes are normalized such that the basis functions form an orthonormal system in both cases, $\Delta x$ being the unit length. The corresponding classic FDTD Yee equations are:

$$
\begin{aligned}
& E_{k+1,2 m}^{Y}=E_{k, 2 m}^{Y}+\frac{2 \Delta t}{\varepsilon \Delta x}\left(H_{k, 2 m}^{Y}-H_{k, 2 m-1}^{Y}\right) \\
& E_{k+1,2 m+1}^{Y}=E_{k, 2 m+1}^{Y}+\frac{2 \Delta t}{\varepsilon \Delta x}\left(H_{k, 2 m+1}^{Y}-H_{k, 2 m}^{Y}\right) \\
& H_{k, 2 m}^{Y}=H_{k-1,2 m}^{Y}+\frac{2 \Delta t}{\mu \Delta x}\left(E_{k, 2 m+1}^{Y}-E_{k, 2 m}^{Y}\right) \\
& H_{k, 2 m+1}^{Y}=H_{k-1,2 m+1}^{Y}+\frac{2 \Delta t}{\mu \Delta x}\left(E_{k, 2 m+2}^{Y}-E_{k, 2 m+1}^{Y}\right)
\end{aligned}
$$


The equivalence between the two systems of equations becomes evident if one replaces

$$
\begin{aligned}
& E_{k, m}^{\Phi^{0}}=\frac{1}{\sqrt{2}}\left(E_{k, 2 m}^{Y}+E_{k, 2 m+1}^{Y}\right) \\
& E_{k, m}^{\Psi^{0}}=\frac{1}{\sqrt{2}}\left(E_{k, 2 m}^{Y}-E_{k, 2 m+1}^{Y}\right) \\
& H_{k, m}^{\Phi^{0}}=\frac{1}{\sqrt{2}}\left(H_{k, 2 m}^{Y}+H_{k, 2 m+1}^{Y}\right) \\
& H_{k, m}^{\Psi^{0}}=\frac{1}{\sqrt{2}}\left(H_{k, 2 m}^{Y}-H_{k, 2 m+1}^{Y}\right)
\end{aligned}
$$

One can notice that the two schemes are also equivalent in terms of memory requirement.

\section{B. One-dimensional MRTD using an arbitrary number of wavelet levels}

In this section we discuss the possibility of generalizing the Haar MRTD algorithm for an arbitrary number of wavelet levels. Clearly, the introduction of a new wavelet level can be analyzed individually by applying the sampling procedure and then writing each MRTD equation explicitly. However, as the number of wavelet levels increases, the calculations become more complicated and the final expressions are difficult to handle for any implementation purpose. This is especially true for two or three-dimensional problems. Therefore, it appears necessary to develop a more general formulation for the Haar MRTD algorithm, which would also allow a more compact representation of the final equations.

Consider equations (8)-(11) describing the one-dimensional MRTD algorithm with one Haar wavelet level. It is easy to see that this set of equations can be equivalently formulated as two matrix equations: 


$$
\begin{gathered}
{\left[\begin{array}{l}
E_{k+1, m}^{\Phi^{0}} \\
E_{k+1, m}^{\Psi^{0}}
\end{array}\right]=\left[\begin{array}{c}
E_{k, m}^{\Phi^{0}} \\
E_{k, m}^{\Psi^{0}}
\end{array}\right]+\frac{\Delta t}{\varepsilon \Delta x}\left[\begin{array}{cccc}
-1 & 1 & 1 & -1 \\
-1 & 1 & 1 & 3
\end{array}\right]\left[\begin{array}{c}
H_{k, m-1}^{\Phi^{0}} \\
H_{k, m}^{\Phi^{0}} \\
H_{k, m-1}^{\Psi^{0}} \\
H_{k, m}^{\Psi^{0}}
\end{array}\right]} \\
{\left[\begin{array}{c}
H_{k, m}^{\Phi^{0}} \\
H_{k, m}^{\Psi^{0}}
\end{array}\right]=\left[\begin{array}{c}
H_{k-1, m}^{\Phi^{0}} \\
H_{k-1, m}^{\Psi^{0}}
\end{array}\right]+\frac{\Delta t}{\mu \Delta x}\left[\begin{array}{cccc}
-1 & 1 & -1 & 1 \\
1 & -1 & -3 & -1
\end{array}\right]\left[\begin{array}{c}
E_{k, m}^{\Phi^{0}} \\
E_{k, m+1}^{\Phi^{0}} \\
E_{k, m}^{\Psi^{0}} \\
E_{k, m+1}^{\Psi^{0}}
\end{array}\right]}
\end{gathered}
$$

Now let us assume that $N$ is the maximum number of wavelet levels used in the scheme throughout the entire computational domain. The electric and magnetic field scaling basis functions must be staggered by $2^{-(N+1)}$ with respect to each other. If a maximum of $L+1$ wavelet levels are used at the point of coordinate $m$, the MRTD equations can be expressed in matrixvector form as following:

$$
\begin{aligned}
& {\left[\mathbf{E}_{k+1, m}^{L+1}\right]=\left[\mathbf{E}_{k, m}^{L+1}\right]+\frac{\Delta t}{\varepsilon \Delta x}\left[\mathbf{A}_{E}^{L+1}\right]\left[\mathbf{H}_{k, m-1, m}^{L+1}\right]} \\
& {\left[\mathbf{H}_{k, m}^{L+1}\right]=\left[\mathbf{H}_{k-1, m}^{L+1}\right]+\frac{\Delta t}{\mu \Delta x}\left[\mathbf{A}_{H}^{L+1}\right]\left[\mathbf{E}_{k, m, m+1}^{L+1}\right]}
\end{aligned}
$$

where

$$
\begin{aligned}
& {\left[\mathbf{E}_{k, m}^{L+1}\right]=\left[\begin{array}{llllllll}
E_{k, m}^{\Phi^{0,0}} & E_{k, m}^{\Psi^{0,0}} & E_{k, m}^{\Psi^{1,0}} & E_{k, m}^{\Psi^{1,1}} & \ldots & E_{k, m}^{\Psi^{L, 0}} & \ldots & E_{k, m}^{\Psi^{L, L^{L}-1}}
\end{array}\right]^{\mathbf{T}}} \\
& {\left[\mathbf{H}_{k, m}^{L+1}\right]=\left[\begin{array}{llllllll}
H_{k, m}^{\Phi^{0,0}} & H_{k, m}^{\Psi^{0,0}} & H_{k, m}^{\Psi^{1,0}} & H_{k, m}^{\Psi^{1,1}} & \ldots & H_{k, m}^{\Psi^{L, 0}} & \ldots & H_{k, m}^{\Psi^{L, L^{L}-1}}
\end{array}\right]^{\mathrm{T}}} \\
& {\left[\mathbf{E}_{k, m, m+1}^{L+1}\right]=\left[\begin{array}{llllllll}
E_{k, m}^{\Phi^{0,0}} & E_{k, m}^{\Phi^{0,1}} & E_{k, m}^{\Psi^{0,0}} & E_{k, m}^{\Psi^{0,1}} & \ldots & E_{k, m}^{\Psi^{L, 0}} & \ldots & E_{k, m}^{\Psi^{L, 2 L+1}-1}
\end{array}\right]^{\mathbf{T}}} \\
& {\left[\mathbf{H}_{k, m-1, m}^{L+1}\right]=\left[\begin{array}{llllllll}
H_{k, m}^{\Phi^{0,-1}} & H_{k, m}^{\Phi^{0,0}} & H_{k, m}^{\Psi^{0,-1}} & H_{k, m}^{\Psi^{0,0}} & \ldots & H_{k, m}^{\Psi^{L,-2^{L}}} & \ldots & H_{k, m}^{\Psi^{L, L^{L}-1}}
\end{array}\right]^{\mathbf{T}}}
\end{aligned}
$$

In the above expressions we introduced the following notations: 


$$
\begin{aligned}
& \Phi_{m}^{n, i}(x)=2^{n / 2} \Phi_{m}^{0}\left(2^{n} x-i\right) \\
& \Psi_{m}^{n, i}(x)=2^{n / 2} \Psi_{m}^{0}\left(2^{n} x-i\right)
\end{aligned}
$$

where $n$ represents the level of the scaling or wavelet function, and $i$ the spatial shift. The matrices $\left[\mathbf{A}_{E}^{L+1}\right]$ and $\left[\mathbf{A}_{H}^{L+1}\right]$ have size $2^{L+1} \times 2^{L+2}$ and their elements are expressed as integrals of the following form:

$$
\int \frac{\partial \Phi, \Psi_{m}^{n, i}(x)}{\partial x} \Phi, \Psi_{m}^{p, j}\left(x \pm 2^{-N-1}\right) d x
$$

where the $\Phi$ or $\Psi$ functions are used as appropriate and $N$ was defined previously. It can be shown that the integrals in (30) can be evaluated in closed-form. More details on the computations can be found in [19].

As an example, we write the matrices $\left[\mathbf{A}_{E}^{L+1}\right]$ and $\left[\mathbf{A}_{H}^{L+1}\right]$ for $L=1$ (two wavelet levels):

$$
\begin{aligned}
{\left[\mathbf{A}_{E}^{2}\right] } & =\left[\begin{array}{cccccccc}
-1 & 1 & 1 & -1 & 0 & \sqrt{2} & 0 & -\sqrt{2} \\
-1 & 1 & 1 & 3 & 0 & \sqrt{2} & -2 \sqrt{2} & \sqrt{2} \\
-\sqrt{2} & \sqrt{2} & \sqrt{2} & \sqrt{2} & 0 & 2 & 6 & 0 \\
0 & 0 & 0 & -2 \sqrt{2} & 0 & 0 & 2 & 6
\end{array}\right] \\
{\left[\mathbf{A}_{H}^{2}\right] } & =\left[\begin{array}{cccccccc}
-1 & 1 & -1 & 1 & -\sqrt{2} & 0 & \sqrt{2} & 0 \\
1 & -1 & -3 & -1 & -\sqrt{2} & 2 \sqrt{2} & -\sqrt{2} & 0 \\
0 & 0 & 2 \sqrt{2} & 0 & -6 & -2 & 0 & 0 \\
\sqrt{2} & -\sqrt{2} & -\sqrt{2} & -\sqrt{2} & 0 & -6 & -2 & 0
\end{array}\right]
\end{aligned}
$$

Extension to two or three dimensions poses no particular problem. In these cases, each spatial derivative that appears in the curl terms is represented by a separate matrix-vector multiplication. The size of these matrices is always dictated by the highest level of wavelets taken into consideration. 


\section{Extension to two dimensions}

The combination of a Haar scaling function and one wavelet level, in two dimensions, is shown in Fig. 4. We consider TE polarization (with the electric field transverse to the propagation plane $x-y$ ). The support of the expansion terms for the three field components, $E_{z}, H_{x}$ and $H_{y}$ are shown in Fig. 5. As an example, we discretize the equation

$$
\frac{\partial H_{y}}{\partial t}=\frac{1}{\mu} \frac{\partial E_{z}}{\partial x}
$$

The field expansion for $E_{z}$ in two dimensions, using one scaling and one level wavelet (Haar basis), can be written as

$$
E_{z}(x, y, t)=\sum_{k, m=-\infty}^{\infty}\left[\begin{array}{l}
E z_{k, m, n}^{\Phi_{x} \Phi_{y}} h_{k}(t) \Phi_{m}(x) \Phi_{n}(y)+E z_{k, m, n}^{\Psi_{x} \Phi_{y}} h_{k}(t) \Psi_{m}(x) \Phi_{n}(y)+ \\
E z_{k, m, n}^{\Phi_{x} \Psi_{y}} h_{k}(t) \Phi_{m}(x) \Psi_{n}(y)+E z_{k, m, n}^{\Psi_{x} \Psi_{y}} h_{k}(t) \Psi_{m}(x) \Psi_{n}(y)
\end{array}\right]
$$

with similar expressions for $H_{x}$ and $H_{y}$. The usual discretization procedure yields the following set of equations (only the $H_{y}$ update equations are given here):

$$
\begin{aligned}
& H y_{k, m, n}^{\Phi_{x} \Phi_{y}}=H y_{k-1, m, n}^{\Phi_{x} \Phi_{y}}+\frac{\Delta t}{\mu \Delta x}\left(E z_{k, m+1, n}^{\Phi_{x} \Phi_{y}}+E z_{k, m+1, n}^{\Psi_{x} \Phi_{y}}-E z_{k, m, n}^{\Phi_{x} \Phi_{y}}-E z_{k, m, n}^{\Psi_{x} \Phi_{y}}\right) \\
& H y_{k, m, n}^{\Phi_{x} \Psi_{y}}=H y_{k-1, m, n}^{\Phi_{x} \Psi_{y}}+\frac{\Delta t}{\mu \Delta x}\left(E z_{k, m+1, n}^{\Phi_{x} \Psi_{y}}+E z_{k, m+1, n}^{\Psi_{x} \Psi_{y}}-E z_{k, m, n}^{\Phi_{x} \Psi_{y}}-E z_{k, m, n}^{\Psi_{x} \Psi_{y}}\right) \\
& H y_{k, m, n}^{\Psi_{x} \Phi_{y}}=H y_{k-1, m, n}^{\Psi_{x} \Phi_{y}}-\frac{\Delta t}{\mu \Delta x}\left(E z_{k, m+1, n}^{\Phi_{x} \Phi_{y}}+E z_{k, m+1, n}^{\Psi_{x} \Phi_{y}}-E z_{k, m, n}^{\Phi_{x} \Phi_{y}}+3 E z_{k, m, n}^{\Psi_{x} \Phi_{y}}\right) \\
& H y_{k, m, n}^{\Psi_{x} \Psi_{y}}=H y_{k-1, m, n}^{\Psi_{x} \Psi_{y}}-\frac{\Delta t}{\mu \Delta x}\left(E z_{k, m+1, n}^{\Phi_{x} \Psi_{y}}+E z_{k, m+1, n}^{\Psi_{x} \Psi_{y}}-E z_{k, m, n}^{\Phi_{x} \Psi_{y}}+3 E z_{k, m, n}^{\Psi_{x} \Psi_{y}}\right)
\end{aligned}
$$




\section{Implementation of the MRTD for Scattering Problems}

\section{A. Implementation of the incident field as a plane wave}

A central issue in modeling an electromagnetic scattering problem with distant sources using MRTD is the capability of implementing the incident field as a plane wave. In this paper, we follow an idea used in traditional FDTD implementations, that is, splitting the computational domain into two regions: one of total fields and the other of scattered fields only, separated by a connecting surface [9]. The incident field is introduced as a 'boundary condition' at this surface, in order to enforce the continuity of the tangential field components. In order to explain the principle of the method, we again use a one-dimensional model, the extension to higher dimensions being straightforward. Also, we consider a field expansion in terms of a Haar scaling function and one Haar wavelet level. Extension to further wavelet levels follows directly.

Fig. 6 illustrates the total and scattered fields regions along the $x$ axis, separated by the 'connecting surfaces' (which in this case are reduced to two points). Consider the 'connecting surface' located at a distance $m \Delta x$ from the left boundary. The electric and magnetic field components at this point depend on field components situated on both sides of the 'connecting surface'. In consequence, the update equations for these components contain both total and scattered field terms (they are labeled with $t$ and $s$, respectively). In order to keep the equations consistent, correction terms representing components of the incident field must be introduced. The following equations are obtained (the label $i$ stands for incident):

$$
\begin{aligned}
& E_{k+1, m}^{\Phi^{0}, t}=E_{k, m}^{\Phi^{0}, t}+\frac{\Delta t}{\varepsilon \Delta x}\left(H_{k, m}^{\Phi^{0}, t}-H_{k, m}^{\Psi^{0}, t}-H_{k, m-1}^{\Phi^{0}, s}+H_{k, m-1}^{\Psi^{0}, s}\right)+\frac{\Delta t}{\varepsilon \Delta x}\left(-H_{k, m-1}^{\Phi^{0}, i}+H_{k, m-1}^{\Psi^{0}, i}\right) \\
& E_{k+1, m}^{\Psi^{0}, t}=E_{k, m}^{\Psi^{0}, t}+\frac{\Delta t}{\varepsilon \Delta x}\left(H_{k, m}^{\Phi^{0}, t}+3 H_{k, m}^{\Psi^{0}, t}-H_{k, m-1}^{\Phi^{0}, s}+H_{k, m-1}^{\Psi^{0}, s}\right)+\frac{\Delta t}{\varepsilon \Delta x}\left(-H_{k, m-1}^{\Phi^{0}, i}+H_{k, m-1}^{\Psi^{0}, i}\right) \\
& H_{k, m-1}^{\Phi^{0}, s}=H_{k-1, m-1}^{\Phi^{0}, s}+\frac{\Delta t}{\mu \Delta x}\left(E_{k, m}^{\Phi^{0}, t}+E_{k, m}^{\Psi^{0}, t}-E_{k, m-1}^{\Phi^{0}, s}-E_{k, m-1}^{\Psi^{0}, s}\right)-\frac{\Delta t}{\mu \Delta x}\left(E_{k, m}^{\Phi^{0}, i}+E_{k, m}^{\Psi^{0}, i}\right)
\end{aligned}
$$




$$
H_{k, m-1}^{\Psi^{0}, s}=H_{k-1, m-1}^{\Psi^{0}, s}+\frac{\Delta t}{\mu \Delta x}\left(-E_{k, m}^{\Phi^{0}, t}-E_{k, m}^{\Psi^{0}, t}+E_{k, m-1}^{\Phi^{0}, s}-3 E_{k, m-1}^{\Psi^{0}, s}\right)-\frac{\Delta t}{\mu \Delta x}\left(-E_{k, m}^{\Phi^{0, i}}-E_{k, m}^{\Psi^{0}, i}\right)
$$

The incident field is known at all time steps, it equal to the total field in the absence of scatterers. Let $f(x)$ be the known variation of the incident electric field along the $x$ axis at time step $k$. Although the exact computation of the coefficients $E_{k, m}^{\Phi^{0}, i}$ and $E_{k, m}^{\Psi^{0}, i}$ would require taking the inner product of $f(x)$ with the respective basis functions, we can take into account the fact that $f(x)$ has a slow variation inside one cell, and make the following approximations (with reference to Fig. 7):

$$
\begin{aligned}
E_{k, m}^{\Phi^{0}, i} & \cong \frac{1}{2}\left(f\left(x_{1}\right)+f\left(x_{2}\right)\right) \\
E_{k, m}^{\Psi^{0}, i} & \cong \frac{1}{2}\left(f\left(x_{1}\right)-f\left(x_{2}\right)\right)
\end{aligned}
$$

Thus, the 'correction terms' due to the incident electric field in the magnetic field update equations become $-\frac{\Delta t}{\mu \Delta x} f\left(x_{1}\right)$ (for $\left.H_{k, m-1}^{\Phi^{0}}\right)$ and $\frac{\Delta t}{\mu \Delta x} f\left(x_{1}\right)$ (for $H_{k, m-1}^{\Psi^{0}}$ ). The 'correction terms' for the electric field update equations have similar expressions. It is worth mentioning that this approach is exactly equivalent to the implementation of the incident field for the classic Yee algorithm on a mesh with half the step size.

The extension to two dimensions is similar. For instance, if $f(x, y)$ is the known variation of the incident electric field at time step $k$, then the expressions of the incident electric field components can be approximated as follows (with reference to Fig. 8):

$$
\begin{aligned}
& E z_{k, m, n}^{\Phi_{x} \Phi_{y}, i} \cong \frac{1}{4}\left(f\left(x_{1}, y_{1}\right)+f\left(x_{2}, y_{1}\right)+f\left(x_{1}, y_{2}\right)+f\left(x_{2}, y_{2}\right)\right) \\
& E z_{k, m, n}^{\Psi_{x} \Phi_{y}, i} \cong \frac{1}{4}\left(f\left(x_{1}, y_{1}\right)-f\left(x_{2}, y_{1}\right)+f\left(x_{1}, y_{2}\right)-f\left(x_{2}, y_{2}\right)\right)
\end{aligned}
$$




$$
\begin{aligned}
& E z_{k, m, n}^{\Phi_{x} \Psi_{y}, i} \cong \frac{1}{4}\left(f\left(x_{1}, y_{1}\right)+f\left(x_{2}, y_{1}\right)-f\left(x_{1}, y_{2}\right)-f\left(x_{2}, y_{2}\right)\right) \\
& E z_{k, m, n}^{\Psi_{x} \Psi_{y}, i} \cong \frac{1}{4}\left(f\left(x_{1}, y_{1}\right)-f\left(x_{2}, y_{1}\right)-f\left(x_{1}, y_{2}\right)+f\left(x_{2}, y_{2}\right)\right)
\end{aligned}
$$

For the two dimensional case, extra care is required at the corners of the connecting surface, where corrections terms from both the horizontal and vertical lines must be taken into consideration.

\section{B. Modeling material inhomogeneities}

In our formulation, the dielectric can be lossy, with the material properties $(\varepsilon$ and $\sigma)$ independent of frequency. Haar scaling functions with a single wavelet level are used in the following derivation. The one-dimensional propagation along the $x$ direction is described by:

$$
\begin{aligned}
& \varepsilon \frac{\partial E_{z}}{\partial t}+\sigma E_{z}=\frac{\partial H_{y}}{\partial x} \\
& \frac{\partial H_{y}}{\partial t}=\frac{1}{\mu} \frac{\partial E_{z}}{\partial x}
\end{aligned}
$$

When we discretize the left hand side of (46), we may obtain cross-terms between the $\Phi$ and $\Psi$ components of the electric fields, due to the presence of the arbitrary functions $\varepsilon(x)$ and $\sigma(x)$. Thus, the equations which update the $\Phi$ and $\Psi$ components of the electric fields for the cell $\mathrm{m}$ become coupled, and they can be written in matrix form as:

$$
\begin{gathered}
\left(\frac{1}{\Delta t}\left[\varepsilon_{m}\right]+\frac{1}{2}\left[\sigma_{m}\right]\right)\left[\begin{array}{l}
E_{k+1, m}^{\Phi} \\
E_{k+1, m}^{\Psi}
\end{array}\right]-\left(\frac{1}{\Delta t}\left[\varepsilon_{m}\right]-\frac{1}{2}\left[\sigma_{m}\right]\right)\left[\begin{array}{l}
E_{k, m}^{\Phi} \\
E_{k, m}^{\Psi}
\end{array}\right]= \\
\frac{1}{\Delta x}\left[\begin{array}{c}
H_{k, m}^{\Phi}-H_{k, m}^{\Psi}-H_{k, m-1}^{\Phi}+H_{k, m-1}^{\Psi} \\
H_{k, m}^{\Phi}+3 H_{k, m}^{\Psi}-H_{k, m-1}^{\Phi}+H_{k, m-1}^{\Psi}
\end{array}\right]
\end{gathered}
$$


where

$$
\begin{array}{r}
{\left[\varepsilon_{m}\right]=\left[\begin{array}{ll}
\varepsilon_{m}^{\Phi \Phi} & \varepsilon_{m}^{\Phi \Psi} \\
\varepsilon_{m}^{\Psi \Phi} & \varepsilon_{m}^{\Psi \Psi}
\end{array}\right]} \\
{\left[\sigma_{m}\right]=\left[\begin{array}{ll}
\sigma_{m}^{\Phi \Phi} & \sigma_{m}^{\Phi \Psi} \\
\sigma_{m}^{\Psi \Phi} & \sigma_{m}^{\Psi \Psi}
\end{array}\right]} \\
\varepsilon, \sigma_{m}^{\Phi \Phi}=\varepsilon, \sigma_{m}^{\Psi \Psi}=\frac{1}{\Delta x} \int_{\text {cell } m} \varepsilon, \sigma(x) d x \\
\varepsilon, \sigma_{m}^{\Phi \Psi}=\varepsilon, \sigma_{m}^{\Psi \Phi}=\frac{1}{\Delta x} \int_{\text {cell } m} \varepsilon, \sigma(x) \Psi_{m}(x) d x
\end{array}
$$

With the notation $\left[M_{m}^{+}\right]=\frac{\Delta x}{\Delta t}\left[\varepsilon_{m}\right]+\frac{\Delta x}{2}\left[\sigma_{m}\right]$ and $\left[M_{m}^{-}\right]=\frac{\Delta x}{\Delta t}\left[\varepsilon_{m}\right]-\frac{\Delta x}{2}\left[\sigma_{m}\right]$, the equation becomes:

$$
\left[\begin{array}{l}
E_{k+1, m}^{\Phi} \\
E_{k+1, m}^{\Psi}
\end{array}\right]=\left[M_{m}^{+}\right]^{-1}\left(\left[M_{m}^{-}\left[\begin{array}{l}
E_{k, m}^{\Phi} \\
E_{k, m}^{\Psi}
\end{array}\right]+\left[\begin{array}{c}
H_{k, m}^{\Phi}-H_{k, m}^{\Psi}-H_{k, m-1}^{\Phi}+H_{k, m-1}^{\Psi} \\
H_{k, m}^{\Phi}+3 H_{k, m}^{\Psi}-H_{k, m-1}^{\Phi}+H_{k, m-1}^{\Psi}
\end{array}\right]\right)\right.
$$

In the two dimensional case, all four components of the electric field may be coupled, and the $\varepsilon$-matrix corresponding to cell $(m, n)$ becomes (the $\sigma$-matrix has similar form)

$$
\left[\varepsilon_{m, n}\right]=\left[\begin{array}{llll}
\varepsilon_{m, n}^{11} & \varepsilon_{m, n}^{12} & \varepsilon_{m, n}^{13} & \varepsilon_{m, n}^{14} \\
\varepsilon_{m, n}^{12} & \varepsilon_{m, n}^{11} & \varepsilon_{m, n}^{14} & \varepsilon_{m, n}^{13} \\
\varepsilon_{m, n}^{13} & \varepsilon_{m, n}^{14} & \varepsilon_{m, n}^{11} & \varepsilon_{m, n}^{12} \\
\varepsilon_{m, n}^{14} & \varepsilon_{m, n}^{13} & \varepsilon_{m, n}^{12} & \varepsilon_{m, n}^{11}
\end{array}\right]
$$

where

$$
\varepsilon_{m, n}^{11}=\frac{1}{\Delta x^{2}} \iint_{\text {cell }(m, n)} \varepsilon(x, y) d x d y
$$




$$
\begin{aligned}
\varepsilon_{m, n}^{12} & =\frac{1}{\Delta x^{2}} \quad \iint_{\text {cell }(m, n)} \varepsilon(x, y) \Psi_{m}(x) d x d y \\
\varepsilon_{m, n}^{13} & =\frac{1}{\Delta x^{2}} \iint_{\text {cell }(m, n)} \varepsilon(x, y) \Psi_{n}(y) d x d y \\
\varepsilon_{m, n}^{14} & =\frac{1}{\Delta x^{2}} \iint_{\text {cell }(m, n)} \varepsilon(x, y) \Psi_{m}(x) \Psi_{n}(y) d x d y
\end{aligned}
$$

Apparently, the formulation presented above complicates the calculations significantly, because for each cell, one needs to compute a number of extra coefficients and invert a matrix. However, the following observations help simplify the task. First, if the medium is homogeneous inside one cell, then the matrices are diagonal and the equations for that particular cell are de-coupled. In consequence, this special kind of update needs to be done only for cells at the boundaries of two or more media. Second, the coefficients of the $\varepsilon$ - and $\sigma$-matrices can be computed for each boundary cell before the time stepping is started, as part of the media configuration generation routine. One can take advantage of the particular form of the $\varepsilon$ - and $\sigma$-matrices, since only four coefficients need to be computed for each of them. These matrices have other nice properties: the product of two such matrices has the same structure and the inverse has the same form as well. It is easy to see that, for any physical media, the matrices $\left[M^{+}\right]$and $\left[M^{-}\right]$are always invertible.

It should be noted, however, that the scheme presented above does not model exactly the electromagnetic fields inside one cell. For instance, it does not enforce the boundary conditions at the interface. Despite this, the method provides increased accuracy as compared with the staircase model of a material interface. It is also interesting to mention that this method is very similar to FDTD models where the media parameters are obtained by averaging across one cell [1]. The approximation is very good as long as the contours of the interface are smooth enough inside the cell. For special configurations involving inhomogeneities smaller than a cell (at least in one direction), the method can produce significant errors, but in that case one can borrow specific techniques developed in the context of FDTD [10]. 


\section{The computational domain}

In this section we indicate the way in which the MRTD Haar wavelet scheme can be implemented in a typical scattering problem. As we mentioned in the Introduction, the multiresolution analysis consists of using high resolution in the regions with fast variation of the fields, while keeping low resolution in other areas where the fields have slow variation [12]. One obvious application of this principle appears when we consider propagation through two media with a high dielectric contrast. Since the wavelength shrinks as the permittivity is increased, finer discretization is needed in the regions with higher dielectric constant. This can be easily achieved by introducing higher-level wavelet functions in the field expansions for those regions, while keeping scaling functions alone for the regions with low dielectric constant (however, even in these regions, the grid size must be reasonably small in order to minimize the dispersion errors). The method is particularly useful when we model scattering in a half-space environment, where in the higher permittivity region we may use one or two levels of wavelet functions, and in air region scaling function alone. Also, any interface between two different media is likely to cause a local increase in the spectral content of the fields, requiring finer resolution in the respective area. In general, higher-level wavelets should be used whenever there is an interface between two media.

In Fig. 9 we illustrate a typical situation, where a dielectric target is placed in a half-space environment above the interface. The shaded areas (the dielectric lower half-space and the area inside and around the target) indicate the use of scaling and wavelet functions, whereas the white areas are covered with scaling functions alone. We have also found that we must use the highest resolution available in the connecting-surface area (and extending to the outer boundaries of the computational domain) in order to preserve the consistency of the scheme throughout the whole domain. The perfectly matched layer (PML) [11] does not present particular problems, because we choose each 'layer' to be one cell thick, inside which the lossy material is homogeneous. 


\section{Numerical Results}

\section{A. Dielectric target in a half-space environment}

The first numerical example demonstrates the benefits of using the Haar MRTD scheme, vis-a-vis the Yee FDTD scheme. The configuration consists of a rectangular dielectric target (conformal to the grid), placed in a half-space environment. All simulations are done in two dimensions. Fig. 10 illustrates the actual computational domain for the MRTD scheme (shaded areas contain scaling plus one level of wavelet functions, whereas white areas contain scaling functions alone). We present a reference solution, which was obtained with the Yee scheme using an appropriate number of grid points per wavelength, uniformly throughout the computational domain. Then we show the solution of the Yee scheme when the number of grid points per wavelength is cut in half, to emphasize the errors introduced by numerical dispersion. We compare these results with those obtained by the MRTD simulation using one level of Haar wavelet functions only in selected regions, while the other areas are covered with scaling functions alone. For the MRTD scheme, we choose a grid step (representative of the scalingfunction support) equal to that of the 'coarse' Yee scheme. Notice that the 'coarse' Yee implementation is equivalent to using scaling functions alone across the whole computational domain (the discussion above concerning decreasing the spatial Yee sampling by a factor of two is equivalent to increasing the scaling-function support by a factor of two).

In addition, this numerical example allows us to compare the Haar-MRTD presented here to that discussed in $[17,18]$. In the implementation presented in $[17,18]$, the update equations for the field components at different resolution levels are decoupled, and, using the analysis in Sec. III.B, it follows that the dielectric boundaries (conformal to the grid) yield no inter-scale coupling. We also note that, using the formulation in $[17,18]$, the incident field implementation yields decoupling between different resolutions. Consequently, for this configuration, the scaling and wavelet components of the field propagate separately, so we cannot exploit the advantage of the multi-resolution approach. To illustrate this point, we model the problem in Fig. 10 by the 
Haar MRTD scheme discussed in [17,18], first using full resolution (scaling plus one-level wavelet functions) across the whole computational domain, then using wavelets only in the regions shaded in Fig. 10. The resulting waveforms are compared with the reference solution.

The reference Yee implementation samples the fields at 20 grid points per smallest wavelength in air (which gives 10 points-per-wavelength inside the dielectric), whereas the 'coarse' Yee implementation uses only 10 grid points per smallest wavelength in air. The MRTD scheme (in both implementations) uses 10 grid points per smallest wavelength in air. By introducing one level Haar wavelets in the shaded areas, the 'effective' sampling rate in these areas is increased to 20 points-per-wavelength. The incident field is implemented as a short pulse plane wave, with the time and frequency domain forms shown in Fig. 11 [21]. We consider TE polarization (as defined in Sec. II.C). The incidence is at $45^{\circ}$ and the observations are made in the far-zone backscatter, in the time-domain. The results are plotted in Fig. 12. First, we notice that using scaling functions alone (Fig. 12a) is not enough for an accurate solution to the problem. The introduction of wavelets in the target region and in areas of higher permittivity yields a very accurate solution (Fig. 12b), and in addition the number of unknowns is reduced via the multi-resolution approach. The results in Fig. 12b use the Haar-MRTD formulation introduced in this paper, and the wavelets are employed as in the shaded regions depicted in Fig. 10 (multiresolution analysis).

We now consider the Haar MRTD scheme introduced in [17,18], in comparison to that developed in this paper (and presented in Fig. 12b). In Figs. 12c and 12d we consider the Haar MRTD scheme introduced in $[17,18]$ for the problem schematized in Fig. 10. In Fig. 12c we consider scaling functions and a single level of wavelets throughout, while in Fig. 12d we consider scaling functions throughout and wavelets as in Fig. 12b (with the goal of a multiresolution analysis). We notice from the solution in Fig. 12c that the MRTD results resemble more the waveform obtained with the 'coarse' FDTD scheme (as in Fig. 12a). Moreover, when attempting the multiresolution analysis (Fig. 12d), the solution deteriorates even further, since the wavelet coefficients cannot propagate over disjoint regions. It is important, 
therefore, to emphasize that the Haar MRTD scheme described in Sec. II allows the application of multi-resolution to a scattering problem, while the formulation discussed in $[17,18]$ does not.

We have presented the results in Fig. 12 to underscore the distinction between the Haar MRTD scheme presented in Sec. II, vis-à-vis that of $[17,18]$. The key point is that if the mesh is conformal with a dielectric target, the method in $[17,18]$ yields no coupling between the scaling and wavelet coefficients, while the method introduced here always has inter-scale coupling. It is important to note, however, that the method in $[17,18]$ does manifest local coupling (at the discontinuity) when the dielectric target is non-conformal. Such coupling is effected through the dielectric-inhomogeneity matrices [18].

\section{B. Two coupled dielectric targets}

As a second example, we consider scattering by two dielectric circular cylinders placed in free-space at a large distance with respect to each other (Fig. 13). This kind of configuration, where relatively small targets are placed at relatively large distances from one another in a homogeneous medium, is particularly suitable for MRTD implementation, because the coverage with high-resolution basis functions is modest compared to the entire computational domain. Therefore, significant savings in terms of computer memory can be obtained as compared to the Yee algorithm using a uniform mesh. For the configuration described in Fig. 13, the memory savings were about $67 \%$ (i.e., MRTD used only one third of the number of unknowns in the FDTD implementation). The simulations are again two-dimensional and most of the parameters of the problem are the same as in Sec. IV.A: same pulse (Fig. 11) as the plane wave excitation, TE polarization, incidence at $45^{\circ}$ and observation in the backscatter. The Yee FDTD scheme utilizes 24 points-per-wavelength in air (at the smallest wavelength), whereas the Haar MRTD scheme samples at 12 points-per-wavelength and uses one level of wavelets only in the target areas (the rest is padded with scaling functions alone). The time step is the same for both schemes. The non-conformal interface was treated according to the formulation in Sec. III.B in the MRTD implementation, and we used a similar approach (averaging the permittivities) for the 
FDTD scheme. As one can see in Fig. 14, the match between the results produced by the two methods is almost perfect, but MRTD has the advantage of using less computational resources. It is interesting to mention that the memory savings would be even more significant in a threedimensional implementation of a similar problem.

\section{Conclusions}

In this paper, the MRTD method has been applied for the first time to electromagnetic scattering problems using a Haar wavelet expansion of the fields. The application of the technique to scattering problems was made possible by a formulation that differs from previous work on Haar-based MRTD schemes, and also by the implementation of the excitation as a plane wave. The benefits of this approach as compared to the classic Yee FDTD have been demonstrated both theoretically and numerically. The multiple resolution capability of the MRTD allows the introduction of higher-resolution basis functions only in the areas where these are needed, while keeping lower resolution for the rest of the computational domain. This may lead to major savings in computational resources, without affecting the accuracy of the results significantly. The algorithm appears robust and relatively simple and there were no signs of numerical instability throughout our simulations.

We believe that the method has great potential in the context of FDTD-related schemes. As future research in this direction, we want to mention the investigation of other wavelet bases for field expansion, which could lead to interesting new results. Also, the application of MRTD to scattering by rough surfaces may prove particularly efficient, because, for this kind of problem, the high resolution needs to be used only in the interface area instead of the whole computational domain.

\section{Acknowledgments}

This research has been supported by Dr. J. Harvey of the US Army Research Office, 
under grant DAAH04-96-1-0448.

\section{References}

[1] A. Taflove, Computational Electrodynamics: The Finite-Difference Time-Domain Method, Artech, Norwood, MA, 1995.

[2] M.F. Hadi and M. Piket-May, "A modified FDTD $(2,4)$ scheme for modeling electrically large structures with high-phase accuracy," IEEE Trans. Antenna and Propagation, vol. 45, pp. 254-264, Feb. 1997.

[3] C.W. Manry, Jr., S.L. Broschat and J.B. Schneider, "Higher order FDTD methods for large problems," ACES J., vol. 10, pp. 17-29, July 1995.

[4] Q. H. Liu, "The PSTD algorithm: a time-domain method requiring only two cells per wavelength," Microwave Opt. Tech. Lett., vol. 15, no.3, pp 158-165, 1997.

[5] Q. H. Liu, "Large-scale simulations of electromagnetic and acoustic measurements using the pseudospectral time-domain (PSTD) algorithm," IEEE Trans. Geoscience and Remote Sensing, vol. 37, pp. 917-926, Mar. 1999.

[6] M. Okoniewski, E. Okoniewska, and M.A. Stuchly., "Three-dimensional subgridding algorithm for FDTD," IEEE Trans. Antennas and Propagation, vol. 45, pp.422-429, Mar. 1997.

[7] M. J. White, M. F. Iskander and Z. Huang, "Development of a multigrid FDTD Code for three-dimensional applications," IEEE Trans. Antennas and Propagation, vol. 45, pp. 15121517, Oct. 1997.

[8] P. Thoma and T. Weiland, "A consistent subgridding scheme for the finite difference time domain method," Int. J. Num. Modeling, vol. 9, pp 359-374, 1996.

[9] K.Umashankar and A. Taflove, "A novel method to analyze electromagnetic scattering of complex objects," IEEE Trans. Electromagn. Compat., vol. 24, pp. 397-405, Nov. 1982.

[10] J. G. Maloney and G. Smith, "The efficient modeling of thin material sheets in the finite difference time domain (FDTD) method, “IEEE Trans. Antenna and Propagation, vol. 40, pp. 323-330, Mar. 1992.

[11] J.P. Berenger, "A perfectly matched layer for the absorption of electromagnetic waves," $J$. Comput. Phys., vol. 114, pp. 185-200, Oct. 1994. 
[12] M. Krumpholz and L. Katehi, "MRTD: New time-domain schemes based on multiresolution analysis," IEEE Trans. Microwave Theory and Techniques, vol. 44, pp. 555-571, Apr. 1996.

[13] R.F. Harrington, Field Computation by Moment Methods, Malabar, FL, Krieger, 1968

[14] M. Krumpholz and P. Russer, "Two-dimensional FDTD and TLM," Int. J. Num. Modeling, vol. 7, pp 141-153, Feb. 1993.

[15] E.T. Tentzeris, R.L. Robertson and L.Katehi, "PML absorbing boundary conditions for the characterization of open microwave circuit components using multiresolution time domain techniques (MRTD)," IEEE Trans. Antennas and Propagation, vol. 47, pp. 1709-1716, Nov. 1999.

[16] E.M. Tentzeris, R.L. Robertson, J. Harvey and L. Katehi, "Stability and dispersion analysis of Battle-Lemarie based MRTD schemes," IEEE Trans. Microwave Theory and Techniques, vol. 47, pp. 1004-1013, July 1999.

[17] K. Goverdhanam, L. Katehi and A. Cangelaris, "Applications of multiresolution based FDTD multigrid," 1997 IEEE MTT-S International Microwave Symposium Digest, pp. 337-340, 1997.

[18] M. Fujii and W.J.R. Hoefer, "A three-dimensional Haar-wavelet-based multiresolution analysis similar to the FDTD method-derivation and application," IEEE Trans. Microwave Theory and Techniques, vol. 46, pp. 2463-2475, Dec. 1998.

[19] T. Dogaru, Modeling and Signal Processing for Electromagnetic Subsurface Sensing, Ph.D. Thesis, Duke University, 1999.

[20] I. Daubechies, Ten lectures on wavelets, SIAM Rev., Philadelphia, PA, 1992

[21] P. Hubral and M. Tygel, “Analysis of the Rayleigh pulse,” Geophysics, vol. 54, pp. 654-658, 1989. 


\section{Figure Captions}

Figure 1. Prototype one dimensional Haar scaling and wavelet functions.

Figure 2. Support of the basis functions in the electric and magnetic field expansions using one level of Haar wavelets (one dimension).

Figure 3. Prototype one dimensional Haar and Yee basis functions.

Figure 4. Prototype two dimensional Haar scaling and wavelet functions.

Figure 5. Support of the basis functions in the electric and magnetic field expansions using one level of Haar wavelets (two dimensions).

Figure 6. Incident field implementation (one dimension).

Figure 7. Approximation of the incident field expansion coefficients (one dimension).

Figure 8. Incident field implementation (two dimensions).

Figure 9. Typical configuration of the computational domain for the MRTD scheme.

Figure 10. Computational domain for the numerical example in Sec. IV.A. The central wavelength is $\lambda_{c}=1 \mathrm{~m}$.

Figure 11. The incident pulse (Rayleigh, $4^{\text {th }}$ order) in (a) time and (b) frequency domain; the central frequency is $300 \mathrm{MHz}$.

Figure 12. Time-domain waveforms for the numerical example in section IV.A. (a) comparison between the reference and the 'coarse' Yee FDTD solution; (b) comparison between the reference and the Haar MRTD scheme described in this paper (Sec. II), with wavelets only in the areas indicated in Fig. 10; (c) comparison between the reference and the Haar MRTD scheme described in [17,18], at full resolution; (d) comparison between the reference and the Haar MRTD scheme described in [17,18], with wavelets only in the areas indicated in Fig. 10.

Figure 13. Computational domain for the numerical example in section IV.B. The central wavelength is $\lambda_{c}=1 \mathrm{~m}$.

Figure 14. Time-domain waveforms for the numerical example described in Fig. 13. 


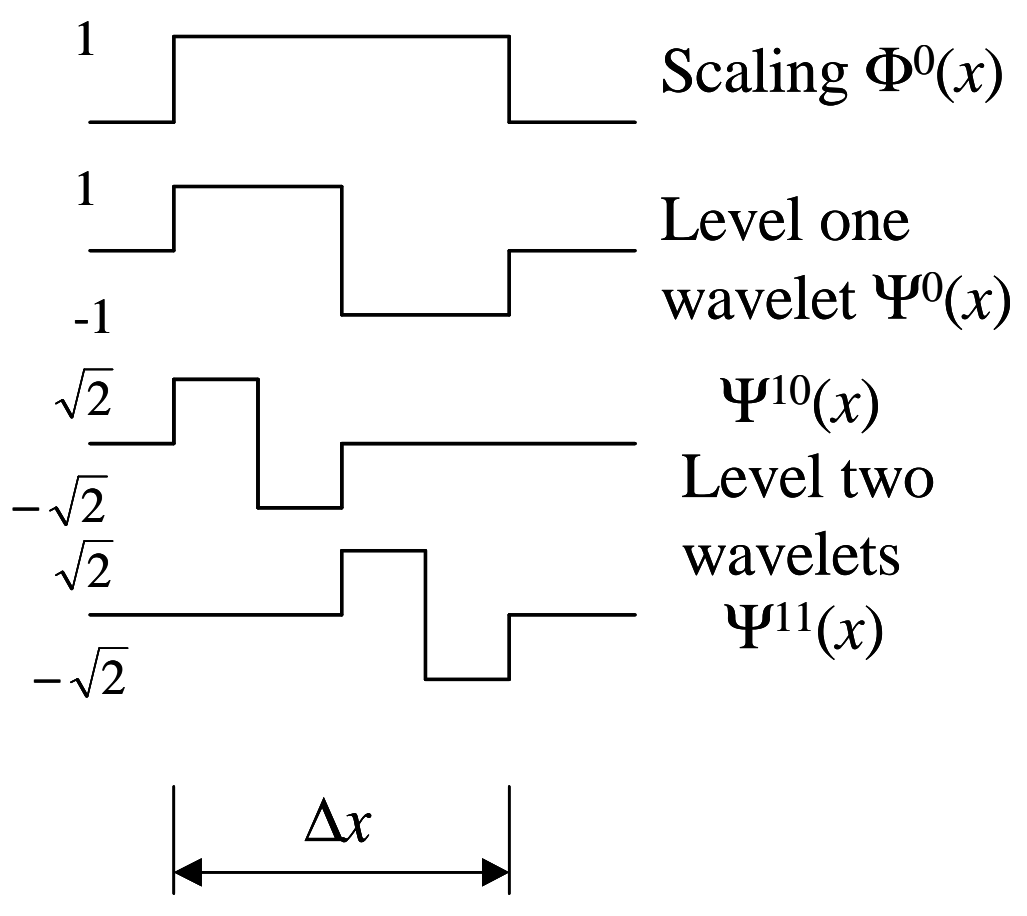

Figure 1

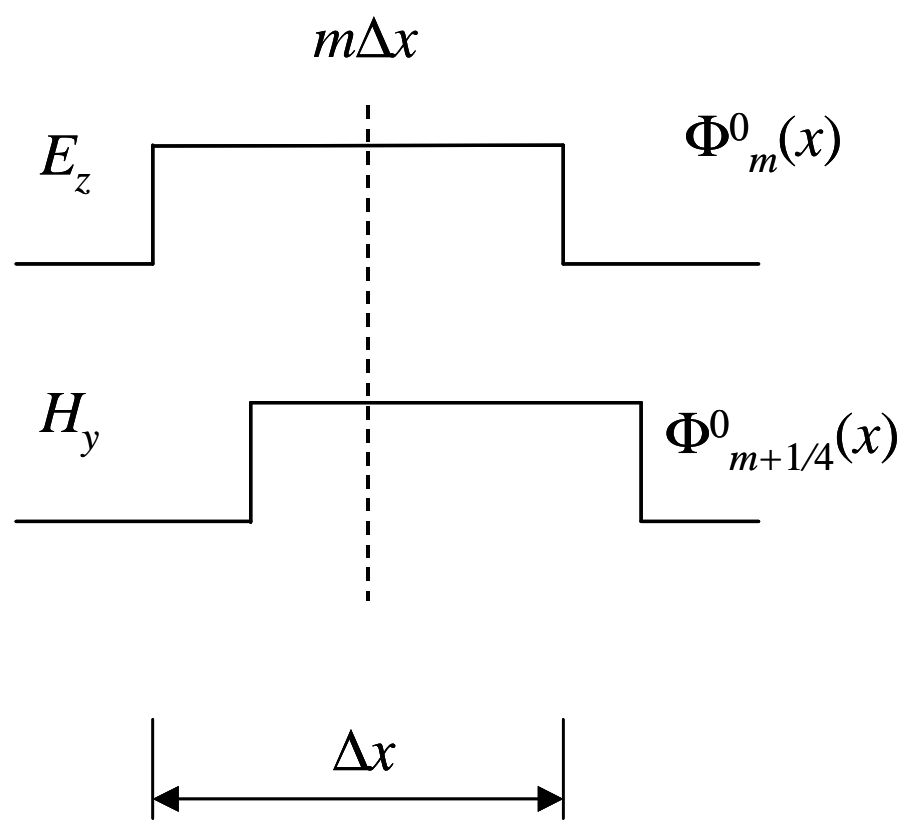

Figure 2 

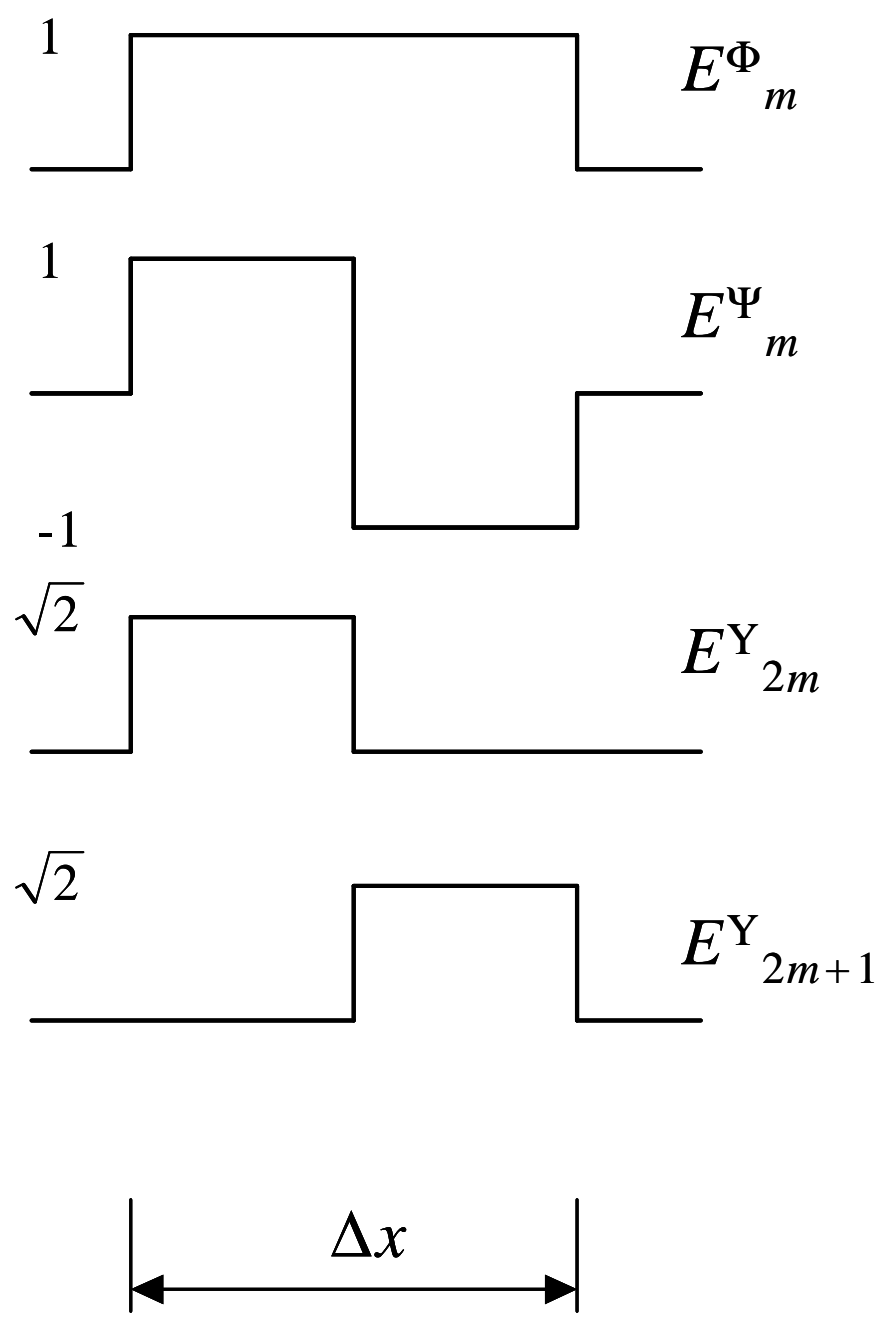

Figure 3 


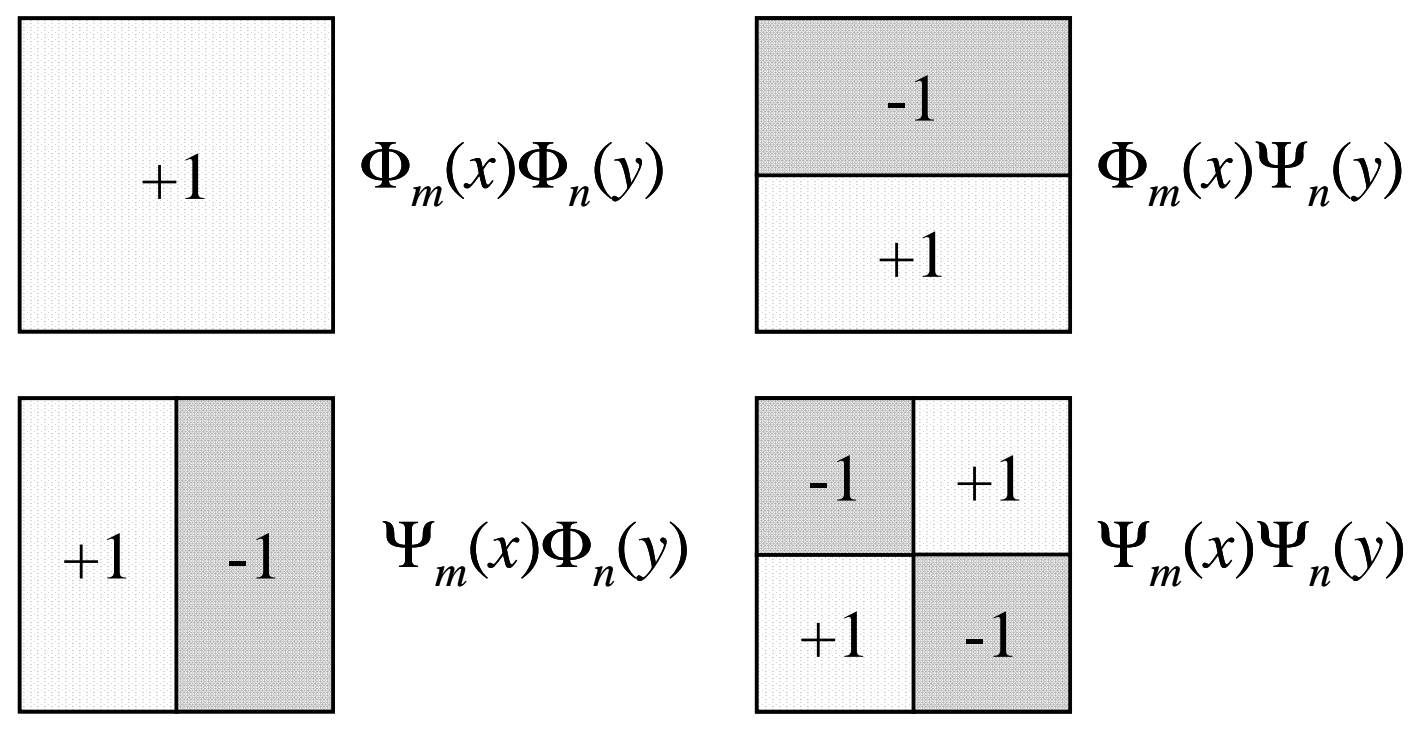

Figure 4

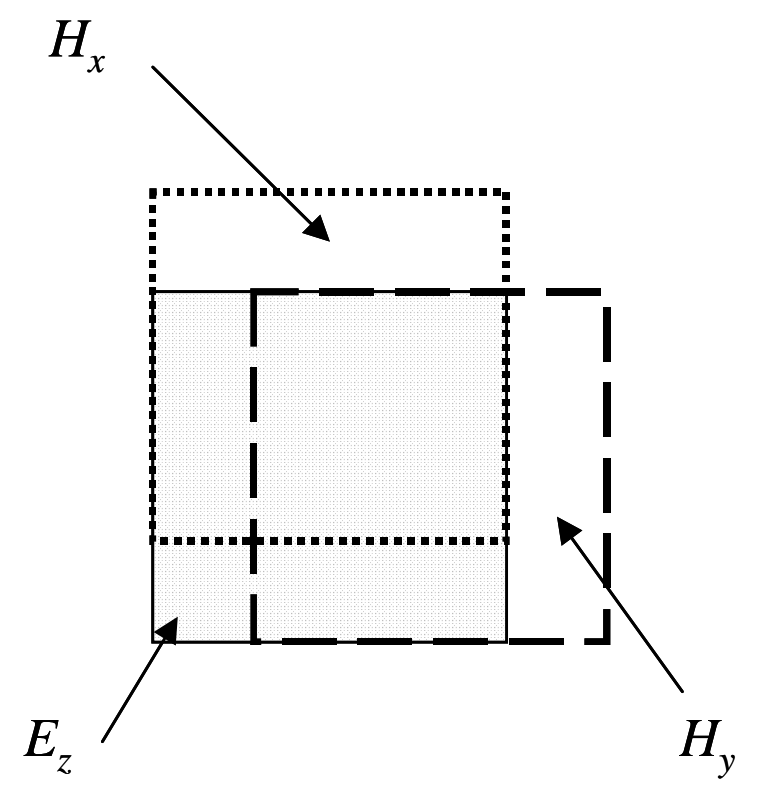

Figure 5 
Scattered fields only

\section{Scattered fields only}

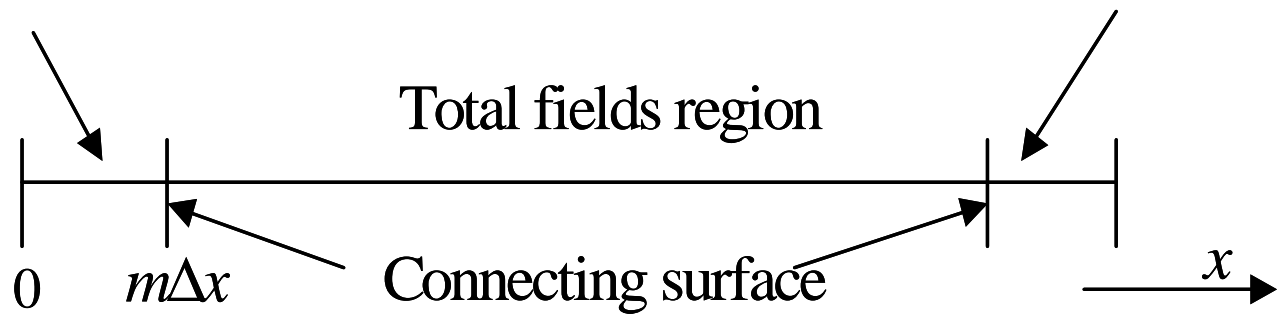

Figure 6

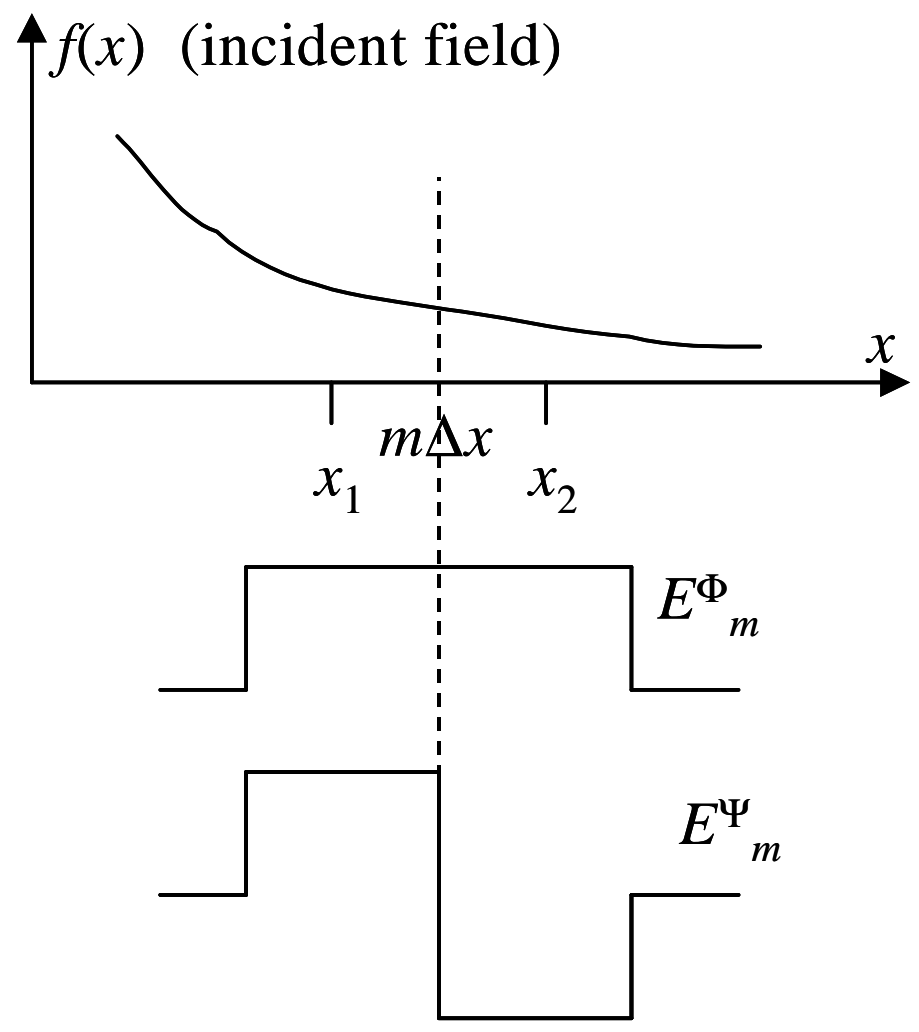

Figure 7 


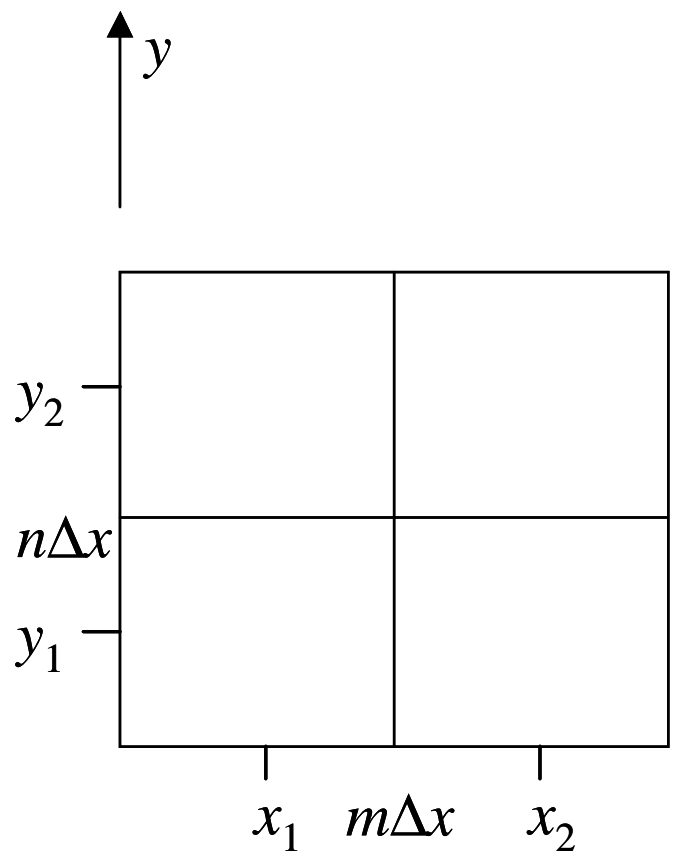

Figure 8

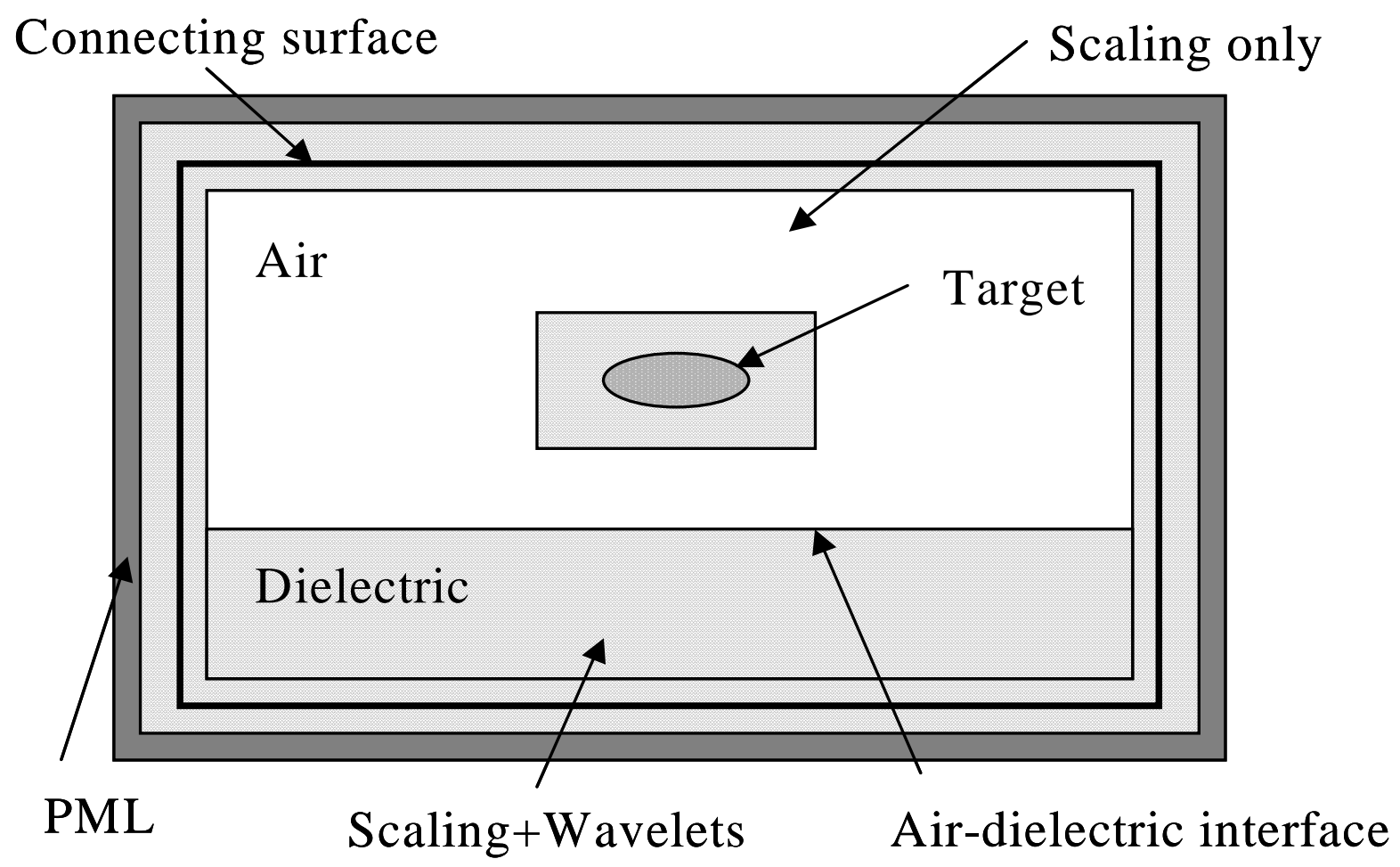

Figure 9 


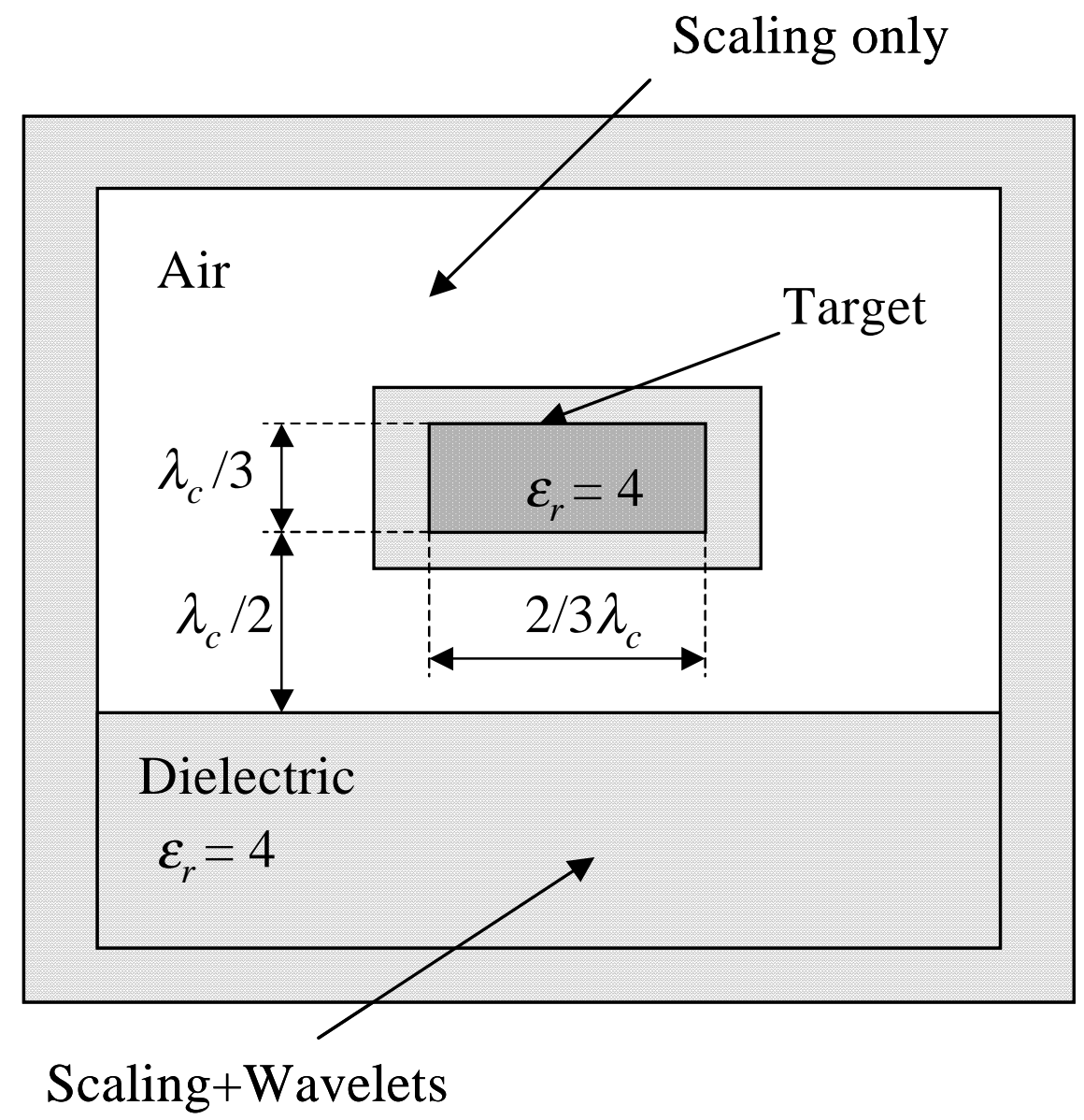

Figure 10 


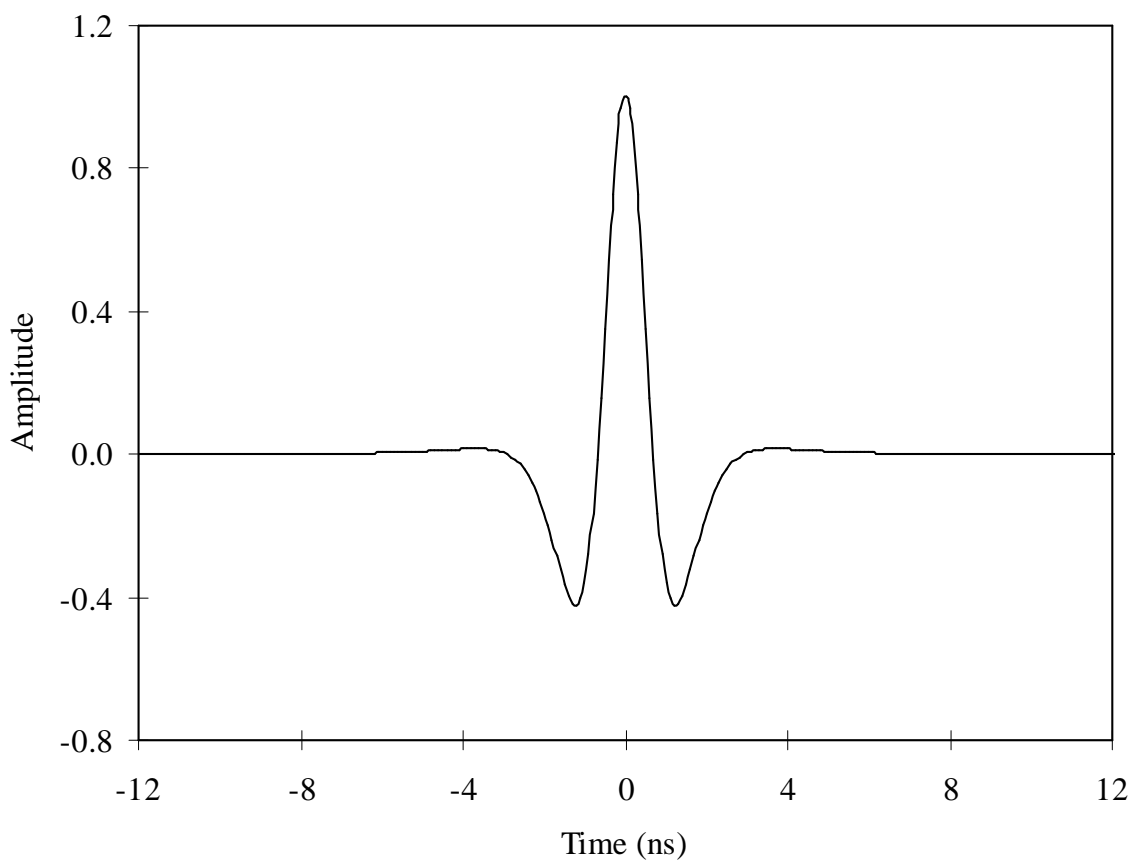

(a)

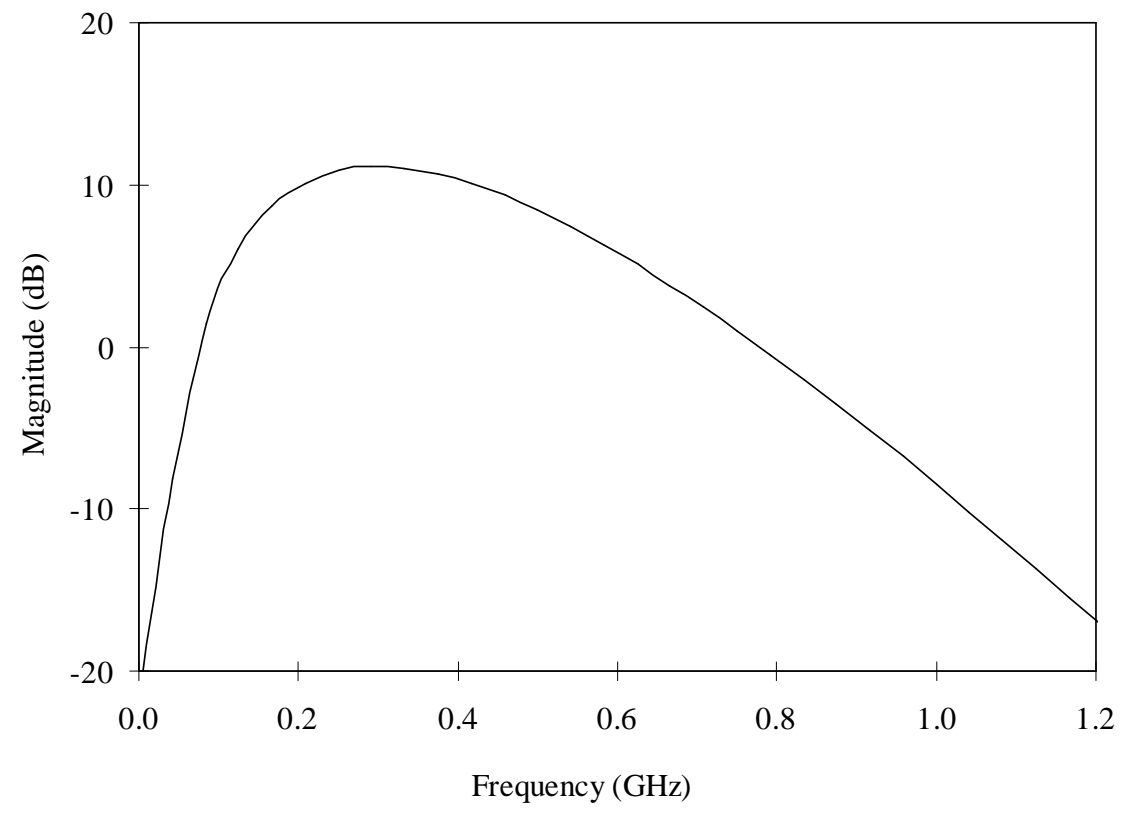

(b)

Figure 11 


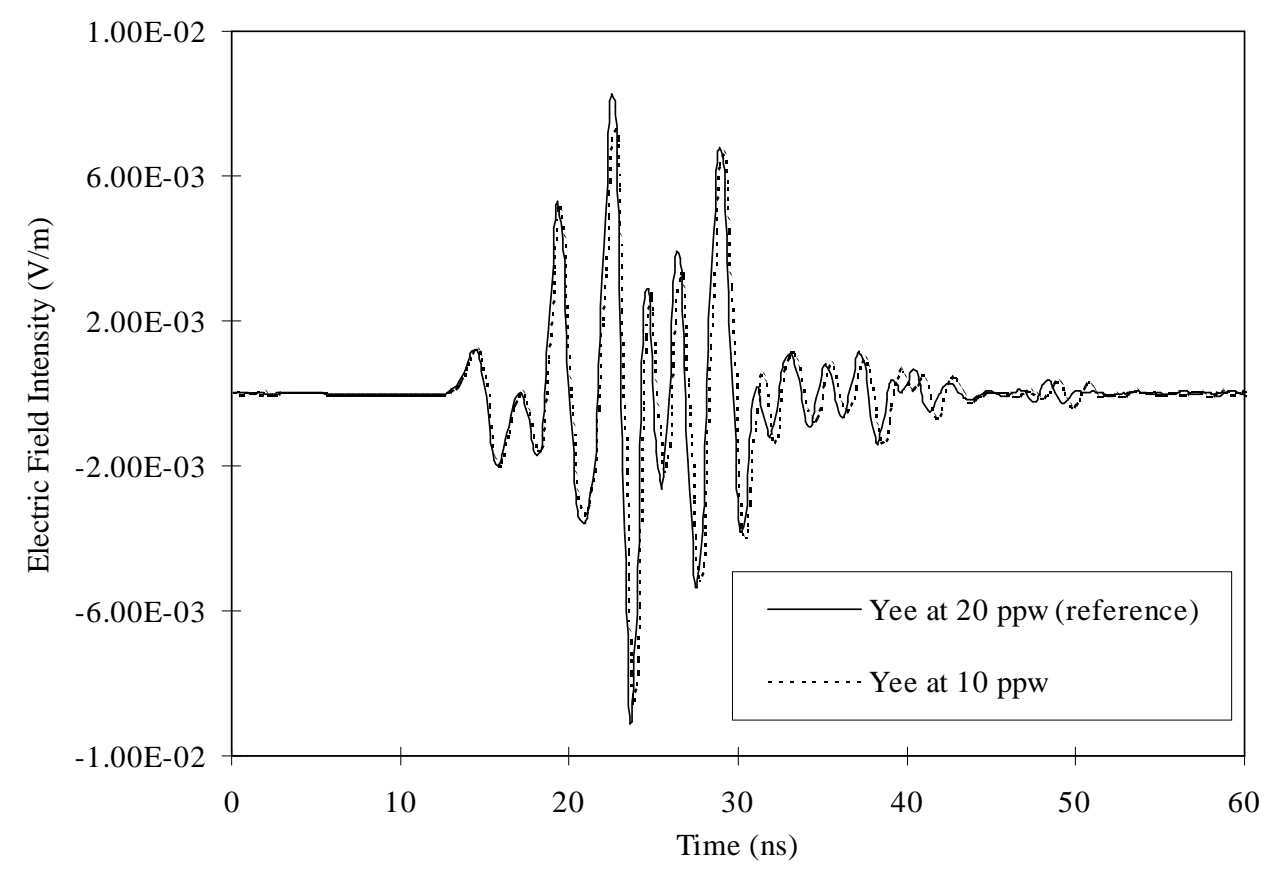

(a)

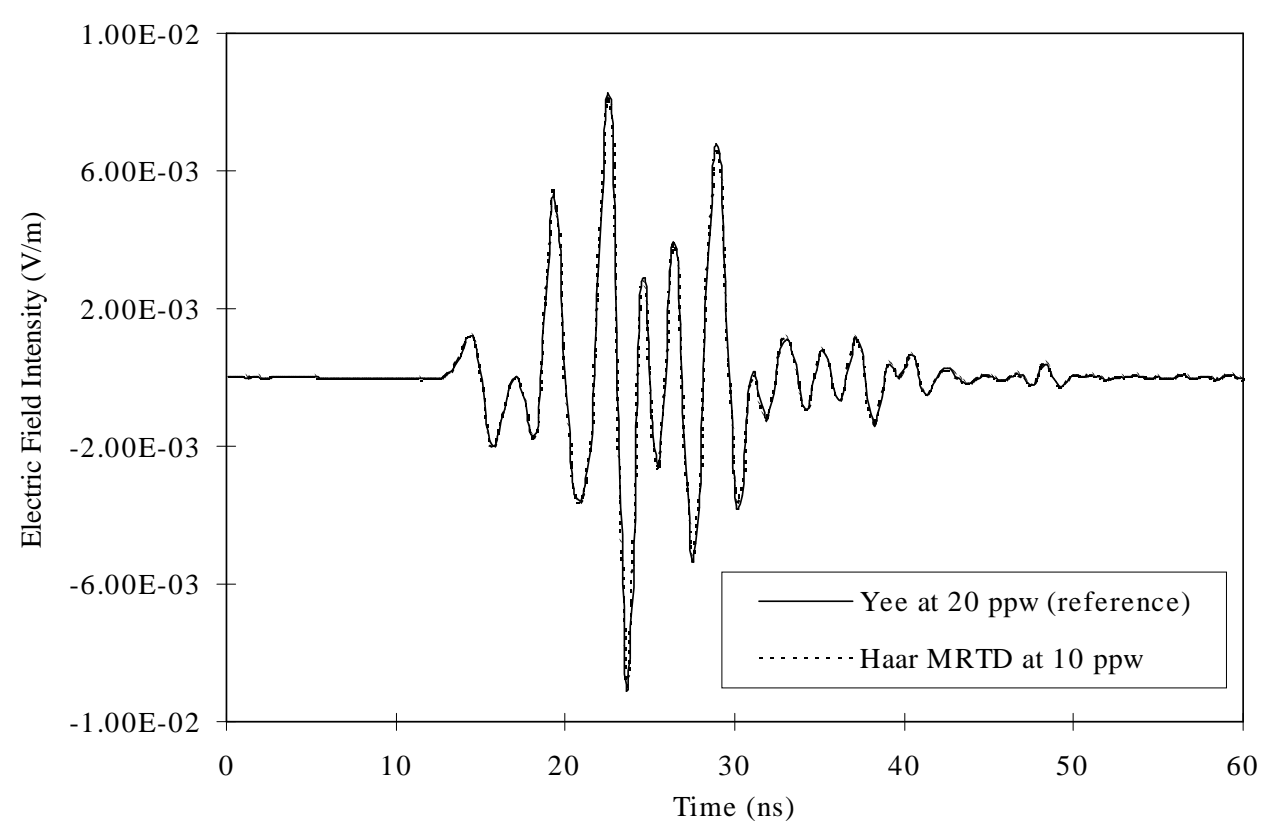

(b)

Figure 12 


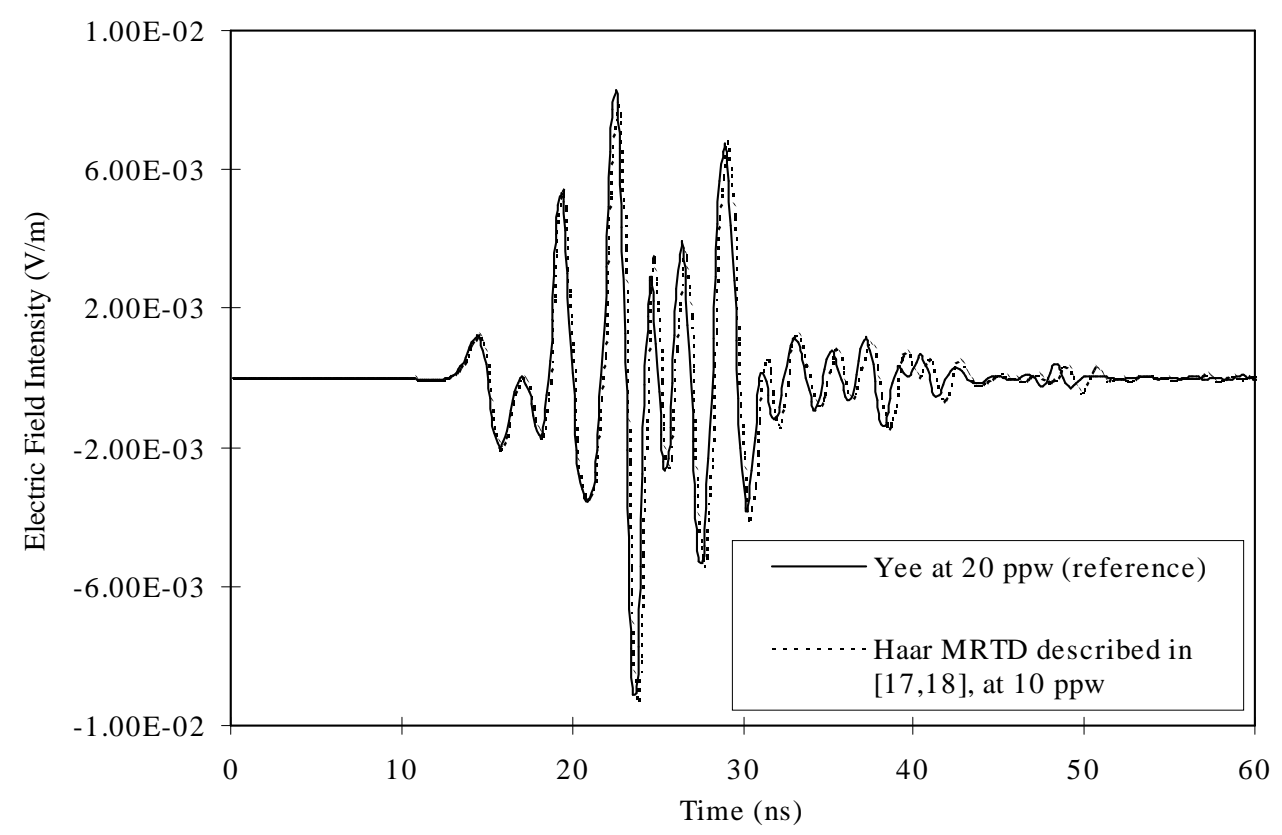

(c)

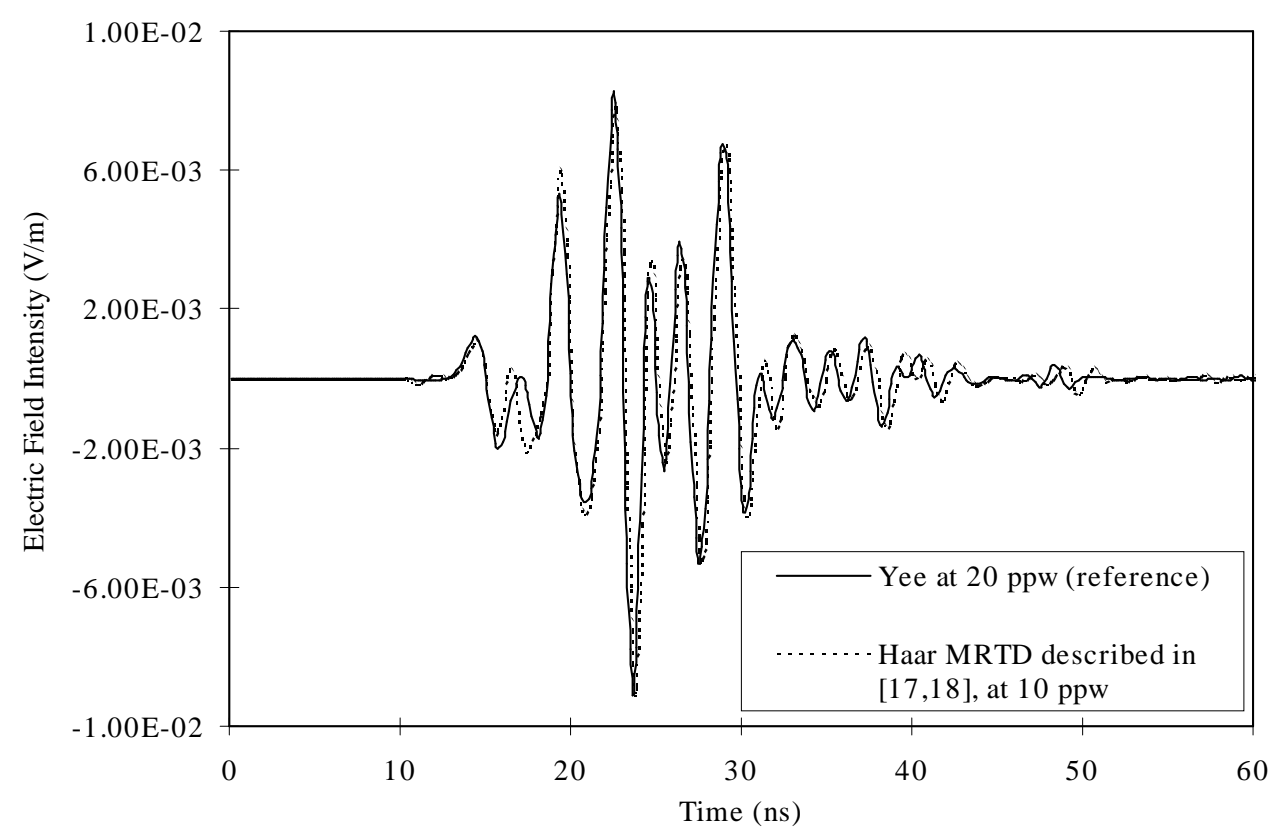

(d)

Figure 12 


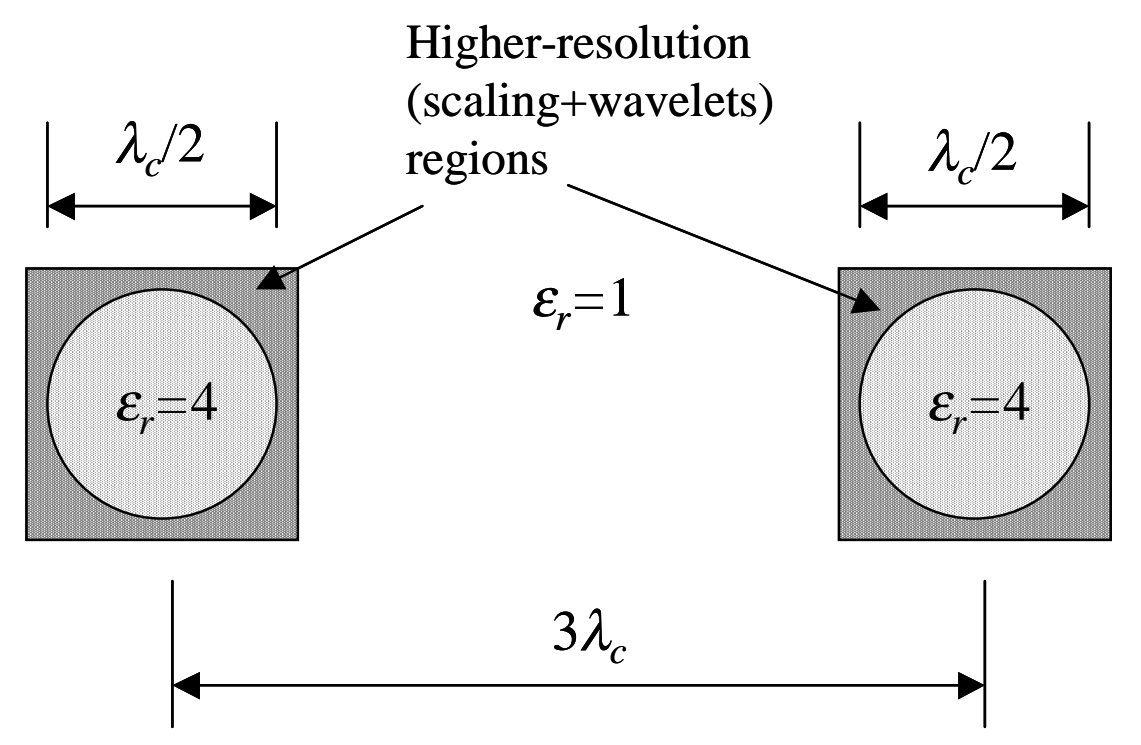

Figure 13

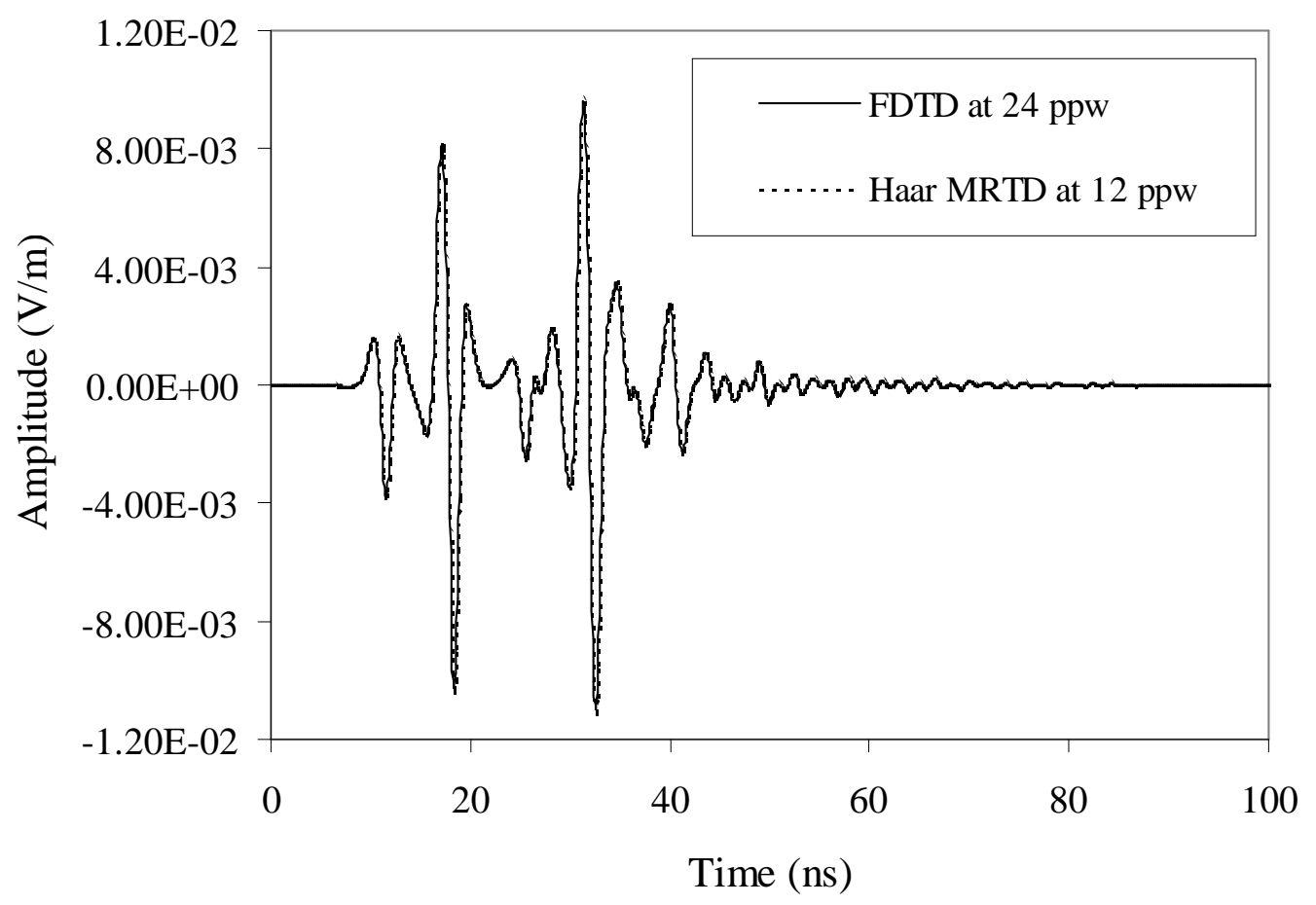

Figure 14 